\title{
Nephrin ectodomain engagement results in Src kinase activation, nephrin phosphorylation, Nck recruitment, and actin polymerization
}

\author{
Rakesh Verma, ${ }^{1}$ lulia Kovari,1 Abdul Soofi, ${ }^{1}$ Deepak Nihalani, ${ }^{1}$ Kevin Patrie, ${ }^{1}$ \\ and Lawrence B. Holzman ${ }^{1,2}$
}

${ }^{1}$ Division of Nephrology, University of Michigan Medical School, Ann Arbor, Michigan, USA. ²Department of Veterans Affairs, Ann Arbor, Michigan, USA.

\begin{abstract}
A properly established and maintained podocyte intercellular junction, or slit diaphragm, is a necessary component of the selective permeability barrier of the kidney glomerulus. The observation that mutation or deletion of the slit diaphragm transmembrane protein nephrin results in failure of podocyte foot process morphogenesis and concomitant proteinuria first suggested the hypothesis that nephrin serves as a component of a signaling complex that directly integrates podocyte junctional integrity with cytoskeletal dynamics. The observations made herein provide the first direct evidence to our knowledge for a phosphorylation-mediated signaling mechanism by which this integrative function is derived. Our data support the model that during podocyte intercellular junction formation, engagement of the nephrin ectodomain induces transient Fyn catalytic activity that results in nephrin phosphorylation on specific nephrin cytoplasmic domain tyrosine residues. We found that this nephrin phosphorylation event resulted in recruitment of the $\mathrm{SH} 2-\mathrm{SH} 3$ domain-containing adapter protein Nck and assembly of actin filaments in an Nck-dependent fashion. Considered in the context of the role of nephrin family proteins in other organisms and the integral relationship of actin dynamics and junction formation, these observations establish a function for nephrin in regulating actin cytoskeletal dynamics.
\end{abstract}

\section{Introduction}

Glomerular visceral epithelial cells play a central role in maintaining the selective filtration barrier of the renal glomerulus. These cells are also termed podocytes to describe the foot-like appearance of numerous interdigitating foot processes that arise from their cell bodies and surround glomerular capillary walls. Glomerular filtrate passes across the specialized intercellular junction - also termed the slit diaphragm - formed at the interface of these interdigitating foot processes.

In response to injury, podocytes undergo a dramatic change in morphology termed foot process effacement resulting from alteration in cytoskeletal and intercellular junctional architecture. By electron microscopy, effacement is manifested by retraction and spreading of podocyte processes. Effacement is a fluid and reversible process that directly correlates with the development of proteinuria both in human disease and in experimental models.

The cellular and molecular mechanisms that integrate podocyte morphology and filter integrity are incompletely defined. Recent investigations have focused on identifying and characterizing the interrelationships and functions of the molecular components of the foot process intercellular junction because several of these components are necessary for development of normal podocyte structure and filter integrity (reviewed in refs. 1, 2).

Nonstandard abbreviations used: CFP, cyan fluorescent protein; 2D, 2-dimensional; DRM, detergent-resistant membrane; GST, glutathione-S-transferase; Nck $\Delta \mathrm{SH} 2$, Nck deleted of its $\mathrm{SH} 2$ domain; nephrinCD, nephrin cytoplasmic domain; P-, phosphorylated; SFK, Src family kinase.

Conflict of interest: The authors have declared that no conflict of interest exists. Citation for this article: J. Clin. Invest. 116:1346-1359 (2006). doi:10.1172/JCI27414.
Nephrin is encoded by NPHS1, the gene mutated in congenital nephrotic syndrome of the Finnish type, a rare autosomal-recessive developmental disorder manifested by failure of foot process morphogenesis (3). At birth, this mutation presents with foot process effacement and heavy proteinuria. Deletion of nephrin by homologous recombination in the mouse results in a similar developmental phenotype (4-6). In the kidney, nephrin is expressed only in podocytes, where it is first expressed at nascent intercellular junctions that form as processes emerge from the basolateral aspect of epithelial podocyte precursors during glomerulogenesis (7). In the mature podocyte, nephrin is targeted to the foot process intercellular junction, where it has been suggested that it contributes to the mechanical filter presumed to function at the slit diaphragm $(7,8)$.

Recent work using genetic experimental approaches in Caenorhabditis elegans and Drosophila melanogaster has suggested that nephrin and its ligand Neph1 function in specifying positional or polarized relationships among cells in complex tissues (9-11); by analogy, this protein complex might function in a similar fashion in the developing podocyte by transducing outside-in signals that integrate junctional and cytoskeletal dynamics and contribute to appropriate tissue morphogenesis. Several observations suggest that nephrin and the complex of proteins with which it is physically associated serve as a signaling nexus that integrate intercellular junction and cytoskeletal dynamics (12). This complex presumably associates with the foot process's subcortical actin cytoskeleton (13) via intermediary proteins that include ZO-1 (14), CD2ap (15), and CASK (16). While it is unlikely that all of the components of the nephrinassociated protein complex have been defined, it is remarkable that deletion in mice of nephrin-interacting proteins including Neph1, 


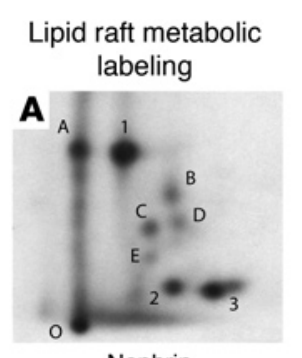

Nephrin

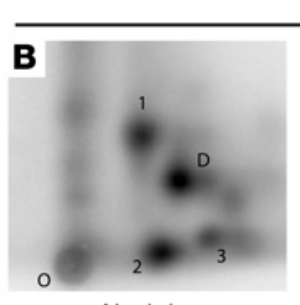

Nephrin
In vitro phosphorylation

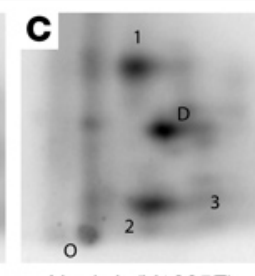

Nephrin(Y1225F)

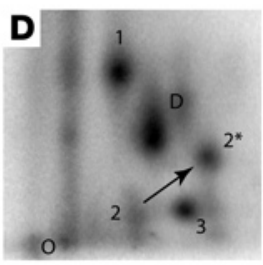

Nephrin(Y1153F)

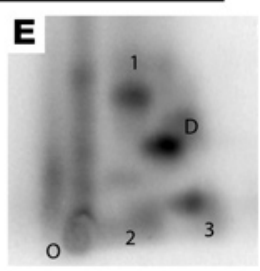

Nephrin(Y1153,1154F)

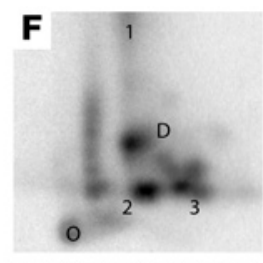

G
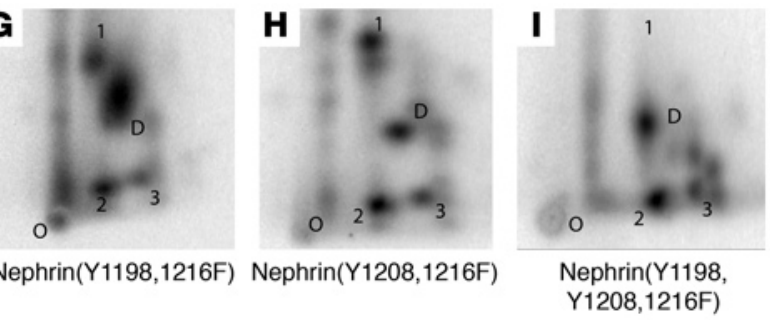

\begin{tabular}{cccc} 
J & Defined & $\begin{array}{c}\text { Podocyte } \\
\text { nephrin } \\
\text { in vivo }\end{array}$ & $\begin{array}{c}\text { rNephrin } \\
\text { in vitro }\end{array}$ \\
\hline 1 & Y1198,Y1208 & + & + \\
2 & Y1153,Y1154 & + & + \\
3 & Y1225 & + & + \\
A & Undefined & + & \\
B & Undefined & + & \\
C & Undefined & + & $?$ \\
D & Undefined & + & \\
E & Undefined & + &
\end{tabular}

\section{Figure 1}

Identification of Fyn-dependent tyrosine phosphorylation sites on nephrin. For in vivo metabolic labeling of nephrin (A), DRM obtained from isolated mouse glomeruli was pelleted, resuspended in kinase buffer, and incubated in the presence of [ $\left.{ }^{32} \mathrm{P}-\gamma\right]$ ATP. These DRM were re-extracted in RIPA buffer, and nephrin was obtained thereafter by immunoprecipitation. For in vitro phosphorylation of nephrin by recombinant Fyn (B-I), purified recombinant GST-nephrinCD or various GST-nephrinCD point mutants were phosphorylated in vitro by recombinant Fyn. Phosphorylated bands representing nephrin protein or its mutants were separated by SDS-PAGE, identified by autoradiography, and processed for 2D tryptic peptide mapping. Representative tryptic peptide maps for radiophosphate-labeled wild type nephrin obtained by both in vivo (A) and in vitro (B) phosphorylation methods are shown juxtaposed for comparison, and corresponding peptides are labeled as defined in $\mathbf{J}$ (along with a summary of peptide characteristics). Mutagenesis of individual tyrosine residues was performed to map Fyn-dependent phosphorylation sites. Where single peptides contained multiple tyrosine residues, single, double, and triple combination tyrosine mutants were created. Shown are representative experiments. Note that for peptide 2, mutation of Y1154 had no effect, Y1153F mutation resulted in a shift in mobility (arrow denotes the shift from 2 to $2^{*}$ ), and mutation of both Y1153 and Y1154 to phenylalanine resulted in disappearance of peptide 2. O, origin; rNephrin, recombinant nephrin.

podocin, and CD2ap results in the development of proteinuria and alteration of podocyte cytoskeletal architecture (15, 17-19).

Nephrin is the target of multiple phosphorylation events (20, 21). At least some of these phosphorylation events are mediated through the Src family kinase (SFK) Fyn (20-22). Fyn resides in podocyte lipid rafts that are specialized cholesterol-rich membrane microdomains in which nephrin, podocin, and Neph 1 are also found (22). Fyn can directly bind to and phosphorylate nephrin in situ, and moreover, Fyn null mice exhibit proteinuria and abnormalities in the development of the foot process intercellular junction (23). Based on these observations, we hypothesized that Fynmediated tyrosine phosphorylation of nephrin initiates signaling events that might dynamically integrate podocyte actin cytoskeleton dynamics and podocyte intercellular junction formation.

\section{Results}

Nephrin is phosphorylated by Fyn on multiple tyrosine residues. Nephrin is tyrosine phosphorylated in situ by the SFK Fyn $(20,21,23)$. In order to study the biological significance of this observation in detail, Fyn-dependent phosphorylated tyrosine (PY) residues were initially mapped. Recombinant glutathione $S$-transferase-nephrin cytoplasmic domain (GST-nephrinCD) expressed in and purified from bacteria was phosphorylated in vitro by recombinant Fyn. NephrinCD phosphorylated in this fashion was isolated and trypsinized, and tryptic peptides were resolved by 2-dimensional (2D) chromatography. Given the presence of 10 tyrosine residues in mouse nephrinCD, it was not unexpected that multiple radiolabeled nephrin tryptic phosphopeptides were observed (Figure 1B). Therefore, systematic mutagenesis of each tyrosine residue located within the nephrinCD was performed to identify Fyn-dependent phosphorylation sites. Where single tryptic peptides contained multiple tyrosine residues, single, double, and triple combination tyrosine mutants were created. Composite analysis allowed the identification of 5 Fyn-dependent PY residues on nephrinCD as summarized in Figure 1J and Table 1. Y1153 and Y1154 were present on the same tryptic peptide, and both were phosphorylated 
Table 1

Fyn-dependent PY mouse residues

\begin{tabular}{ccccc} 
Residue & Sequence & \multicolumn{3}{c}{ Characterization } \\
& & $\begin{array}{c}\text { Mutagenesis and } \\
\text { 2D mapping }\end{array}$ & PY Ab & Li et al. (20) \\
Y1128 & YEES & Inconclusive & Not tested & Not tested \\
Y1153 & YYSM & Yes & Not tested & Inconclusive \\
Y1154 & YYSM & Yes & Not tested & Not tested \\
Y1172 & YRQA & Inconclusive & Not tested & Yes \\
Y1191 & YDEV & Inconclusive & Yes & Not tested \\
Y1198 & YGPP & Yes & Not tested & Not tested \\
Y1208 & YDEV & Yes & Yes & Yes \\
Y1216 & YDLR & Inconclusive & Not tested & Not tested \\
Y1225 & YEDP & Yes & Not tested & Not tested \\
Y1232 & YDQV & Inconclusive & Not tested & Inconclusive
\end{tabular}

by Fyn. Similarly, Y1198, Y1208, and Y1218 were located on the same tryptic fragment; however, only Y1198 and Y1208 were phosphorylated by Fyn.

Since nephrin and Fyn reside in lipid rafts (Triton X-100insoluble membrane fraction, or detergent-resistant membrane [DRM] in podocyte foot processes, we sought to confirm that the pattern of nephrin tyrosine residue phosphorylation identified in vitro occurred in situ in a similar fashion. For this purpose, DRM obtained by flotation gradient centrifugation from isolated mouse glomeruli was resuspended and incubated in a kinase buffer containing [ $\left.{ }^{32} \mathrm{P}-\gamma\right]$ ATP. This DRM was re-extracted with RIPA buffer, and nephrin was immunoprecipitated and isolated before analysis by tryptic phosphopeptide 2D mapping. Comparison of the mobility of tryptic phosphopeptides obtained by this approach to that obtained using phosphorylated recombinant nephrinCD showed similar mobility of peptides in which Fyndependent PY residues had been identified (Figure 1, A and B). It was concluded that mouse nephrin tyrosine residues 1153, 1154, 1198, 1208, and 1225 were phosphorylated by Fyn. Phosphorylation of additional tyrosine residues in the nephrinCD by Fyn or yet unidentified additional protein kinases is possible but could not be confirmed by this analysis.

Development and characterization of a phosphospecific nephrin antibody. Primary sequence analysis revealed that Y1208 sits within a YDEV motif typical of a functional SH2-binding domain. This site is conserved among mouse, rat, and human species. To develop a reagent that would be useful for investigating nephrin phosphorylation-mediated signaling that might involve this domain, an affinity-purified rabbit polyclonal serum was raised against a 10 -residue mouse nephrin phosphopeptide as described in Methods. As shown in Figure 2, this antibody was useful in both immunoblotting and in immunohistochemistry assays. In immunoblotting experiments, anti-P-nephrin antibody specifically recognized nephrin expressed in COS7 cells or endogenous nephrin extracted from isolated mouse glomeruli only after the cells or kidney were pretreated with pervanadate, a nonspecific protein phosphatase inhibitor (Figure 2, A and B). A similar result was obtained by indirect immunofluorescence (Figure 2E); here, anti-P-nephrin antibody identified glomeruli in paraffin-embedded adult mouse kidney sections only after kidneys were perfused with pervanadate prior to harvest.

While mouse nephrin Y1191 had not been identified as being phosphorylated by Fyn in the experiments described above, this tyrosine residue resides within a YDEV motif identical to that of Y1208. Therefore, to confirm the epitope specificity of this antibody, plasmids encoding wild-type nephrin or nephrin(Y1191F), nephrin(Y1208F), or nephrin(Y1191/1208F) mutants were expressed in COS7 cells that were or were not treated with pervanadate prior to cell lysis (Figure 2C). Using immunoblotting, P-nephrin antibody identified wild-type nephrin, nephrin(Y1191F), and nephrin(Y1208F), but not the nephrin(Y1191/1208F) double mutant, when cells were pretreated with pervanadate prior to lysis. This result suggests both that Y1191 and Y1208 become phosphorylated in the presence of pervanadate and that the anti-P-nephrin antibody recognizes epitopes created when either of these tyrosine residues become phosphorylated. A similar set of experiments demonstrated that transient transfection of SYF cells (mouse embryonic fibroblasts deleted of c-Src, Fyn, and Yes) with plasmid encoding Fyn and nephrin resulted in nephrin phosphorylation at both Y1191 and Y1208 (Figure 2D). These experiments confirm that nephrin can be phosphorylated in cells by Fyn at Y1208 as well as that nephrin can be phosphorylated by Fyn at Y1191.

Nephrin is tyrosine phosphorylated in an SFK-dependent fashion on Y1191 and Y1208 upon engagement of its ectodomain. As has been demonstrated in other membrane receptor complexes that reside in lipid rafts (24), it was hypothesized that engagement of the nephrin ectodomain would result in both activation of a SFK and phosphorylation of nephrin at Y1191 and Y1208. A cell culture model originally described by Lahdenpera et al. was employed to test this hypothesis (21). For these experiments, a monoclonal antibody $(50 \mathrm{~A} 9 \mathrm{mAb})$ directed against the ectodomain of human nephrin or a nonspecific antibody of the same species, and isotype was added to the culture medium of HEK 293 cells that had been stably transfected with human nephrin (Figure 3). Because a phosphospecific pan-Src(Y418) antibody that recognizes the autophosphorylation site within the catalytic domain of SFKs had been previously shown to correlate with SFK activity (25), this antibody was used to assay for induction of SFK activity. Indeed, engagement of the human nephrin ectodomain with the $50 \mathrm{~A} 9 \mathrm{mAb}$ - but not control IgG - resulted in induction of SFK activity (Figure 3A). Concomitantly, nephrin phosphorylation at Y1191 and Y1208 (Figure 3C) occurred within 2 minutes after engagement of the nephrin ectodomain by addition of 50A9 (Figure 3B). This nephrin phosphorylation event was inhibited in the presence of PP2, a SFK-specific inhibitor. Together, these results suggest that engagement of the nephrin ectodomain results sequentially in SFK induction followed by nephrin phosphorylation within $2 \mathrm{SH} 2$-binding domains at mouse nephrin Y1191 and Y1208.

Nephrin tyrosine phosphorylation occurs transiently during podocyte differentiation and in response to podocyte injury. Using indirect immunofluorescence microscopy, the P-nephrin antibody was applied to newborn and adult mouse kidney sections to evaluate when and where nephrin tyrosine phosphorylation occurs in situ under physiological conditions. Foot process morphogenesis begins during the early capillary loop stage of glomerulogenesis when nephrin is first expressed on the tips of nascent budding processes that extend from the lateral walls of undifferentiated podocytes immediately adjacent to the glomerular basement membrane (7). Consistent with the localization of nephrin in podocytes during this process, P-nephrin antibody reacted with newborn kidney along a fine line at the basal aspect of the podocyte. P-nephrin antibody immunoreactivity was first observed at the early capillary 
A

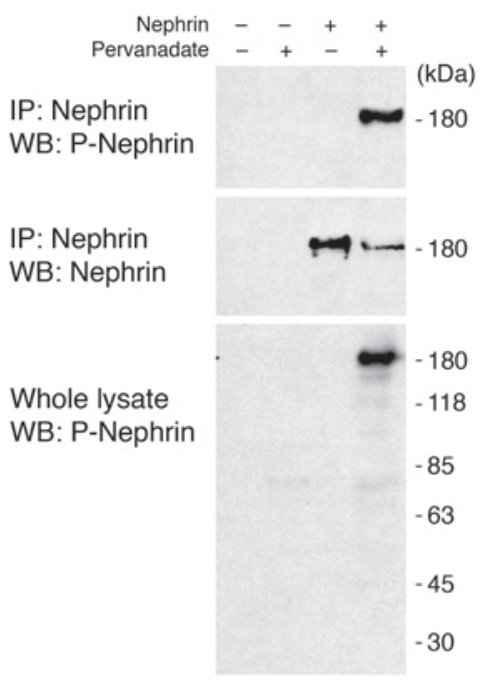

B

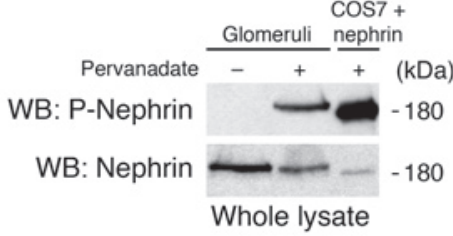

C

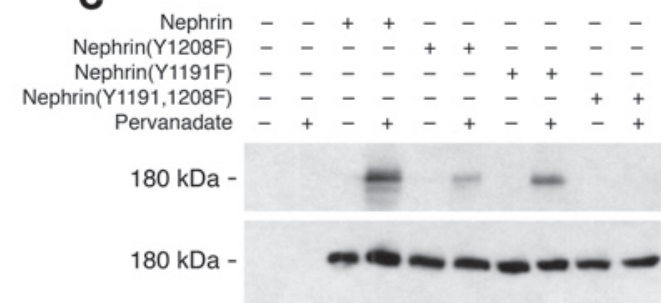

D

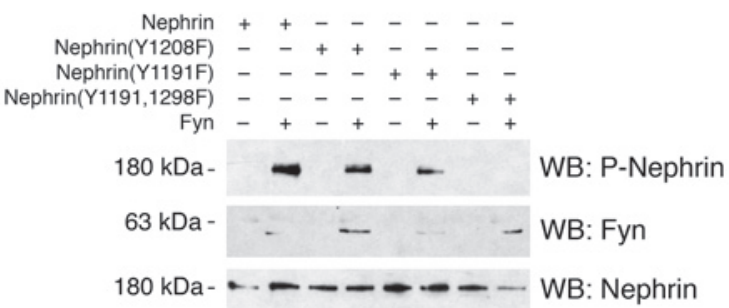

E

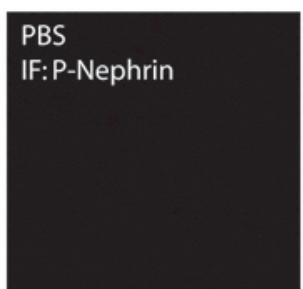

Pervanadate

IF:P-Nephrin
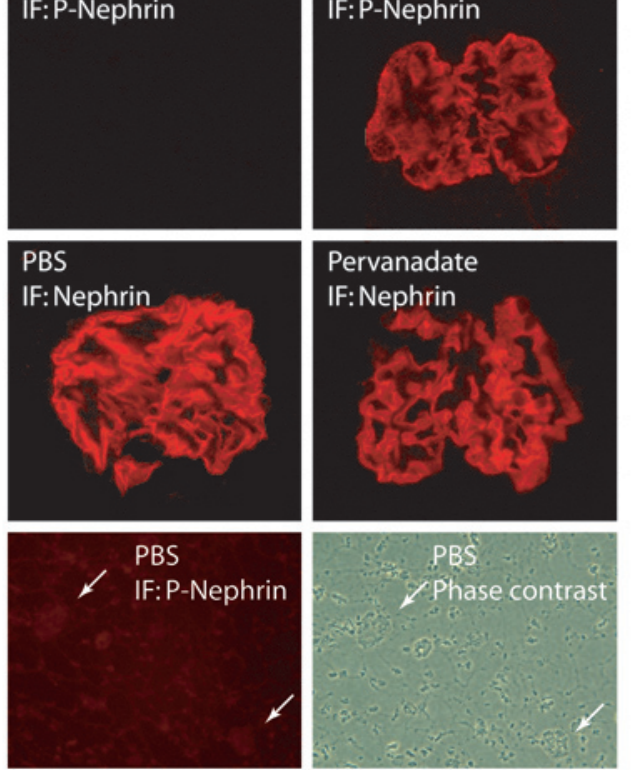

\section{Figure 2}

Characterization of a P-nephrin polyclonal antibody. (A) Cell lysate - obtained from COS7 cells transfected as labeled with plasmid encoding mouse nephrin and treated with or without pervanadate - was analyzed as indicated by immunoblotting with affinity-purified rabbit polyclonal anti-P-nephrin. (B) Mouse kidney was perfused with pervanadate or PBS; subsequent to glomerular isolation and extraction, glomerular lysate was resolved by SDS-PAGE and immunoblotted for P-nephrin. (C) SYF mouse embryonic fibroblasts were transiently transfected with plasmid encoding nephrin or the indicated nephrin mutants and treated or not treated with pervanadate prior to cell lysis. Cell lysates were resolved by SDS-PAGE and immunoblotted with indicated antibodies. (D) COS7 cells were transiently transfected with plasmids encoding nephrin or the indicated nephrin mutants and with Fyn or a control plasmid. Obtained cell lysates were resolved by SDS-PAGE and immunoblotted with indicated antibodies. (E) Top panels: Adult mouse kidneys were perfused with PBS or pervanadate and were subsequently examined by indirect immunofluorescence (IF) using the indicated primary antibodies. Bottom panels: Indirect immunofluorescence microscopy and phase-contrast microscopy of identical fields was used to confirm absence of P-nephrin staining in glomeruli (see Figure 4). Magnification, $\times 40$ (top 4 panels); $\times 20$ (bottom 2 panels).

loop stage. This expression was observed later than initial expression of podocalyxin, which arose on the apical aspect of podocyte precursors late in the S-shaped body phase (Figure 4). Importantly, phosphorylation of mouse nephrin at Y1191 and Y1208 was transient in kidney, since P-nephrin antibody did not react with adult kidney sections except when kidney was perfused before fixation with pervanadate (Figure 2E and Figures 4 and 5).

We speculated that the nephrin membrane protein complex participates in phosphorylation-mediated signal transduction necessary for coordinating intercellular junction formation and podocyte cytoskeletal dynamics. For this reason, it was hypothesized that podocyte injury that results in alterations in cytoskeletal dynamics would induce nephrin tyrosine phosphorylation. The rodent protamine sulfate-heparin sulfate model was employed in mice to investigate this hypothesis (26). In this model, perfusion of mouse kidneys with protamine sulfate results in foot process effacement, while subsequent perfusion with heparin sulfate results largely in restoration of normal podocyte morphology. Adult mouse kidneys were perfused with PBS, protamine sulfate, or protamine sulfate followed by heparin sulfate prior to fixation and analysis by electron microscopy or indirect immunofluorescence microscopy (Figure 5). Control kidneys perfused with PBS exhibited podocytes with normal morphology, and, as observed earlier, control adult mouse kidneys did not react with P-nephrin antibody. Perfusion with protamine sulfate for 15 minutes induced diffuse foot process effacement and resulted in induction of P-nephrin immunoreactivity in a glomerular pattern. Remarkably, perfusion of kidneys with protamine sulfate for 15 minutes followed by perfusion with 

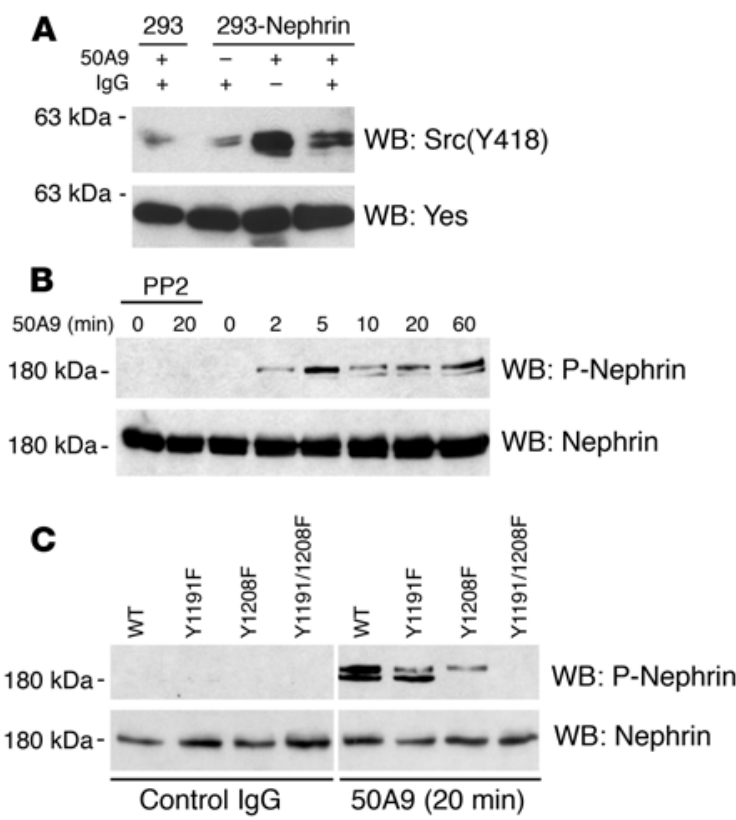

heparin sulfate for 15 minutes largely restored normal foot process morphology and resulted in markedly decreased immunoreactivity to $\mathrm{P}$-nephrin relative to that observed after perfusion with protamine sulfate alone. These experiments were repeated 3 times with similar results. Together these results imply that nephrin tyrosine phosphorylation on Y1191 and Y1208 is induced transiently during podocyte effacement and that restoration of normal podocyte morphology results in attenuation of nephrin phosphorylation at these tyrosine residues.

Identification of SH2 domain-bearing proteins that associate with nephrin in a tyrosine phosphorylation-dependent fashion. To begin to understand the functional significance of nephrin tyrosine phosphorylation, we sought to identify proteins that associate with nephrin in a tyrosine phosphorylation-dependent fashion. A pull-down strategy was devised for this purpose. Mouse nephrinCD was expressed as a GST fusion protein (GST-nephrinCD) in BL21 or TKB1 E. coli cells. TKB1 cells express a thymidine protein tyrosine kinase. For that reason, when transformed with the relevant plasmid, TKB1 cells produced GST-nephrinCD that was tyrosine phosphorylated on Y1208 and on other nephrin tyrosine residues (Figure 6A). To identify P-nephrin-associated proteins, this purified tyrosine-phosphorylated GST-nephrinCD bound to glutathione agarose was mixed with lysate obtained from isolated mouse glomeruli. The obtained purified complex was resolved by SDS-PAGE and immunoblotted with a panel of antibodies for proteins that contain $\mathrm{SH} 2$

\section{Figure 4}

Endogenous nephrin is recognized by P-nephrin antibody in developing but not adult glomeruli. Newborn or adult mouse kidney sections were prepared as described in Methods. Sections were incubated simultaneously with rabbit P-nephrin or total nephrin antibody and with mouse anti-podocalyxin antibody (Podoclx) and were analyzed by indirect immunofluorescence microscopy. Magnification, $\times 400$.

\section{Figure 3}

Engagement of nephrin extracellular domain results in activation of $\mathrm{Src}$ and Fyn-dependent nephrin phosphorylation on Y1191 and Y1208. HEK 293T cells were stably transfected with plasmids encoding human nephrin or nephrin mutants Y1191F, Y1208F, and Y1191/1208F. Cultured transfectants were stimulated where indicated by addition of mouse anti-human nephrin extracellular domain monoclonal antibody (50A9) or with a mouse IgG control antibody of the same isotype and a secondary anti-mouse IgG. (A) Cells were stimulated for 10 minutes by addition of indicated antibody to culture media and were analyzed by immunoblotting with phosphospecific pan-Src(Y418) antibody or an anti-Yes antibody to demonstrate equivalent loading. (B) Time-course experiment demonstrated induction of nephrin tyrosine phosphorylation upon addition of 50A9 antibody to media. Where indicated, cells were pretreated with PP2 for 15 minutes prior to addition of antibodies. Lysates were immunoblotted with P-nephrin or total nephrin antibodies. (C) Indicated stably transfected 293T cells were stimulated for 10 minutes by addition of indicated antibody to culture media and were analyzed by immunoblotting with the indicated antibodies.

domains (Table 2). Nonphosphorylated GST-nephrinCD served as a negative control. Using this approach, GST-nephrinCD associated with Crk, ShcA, and Nck in a tyrosine phosphorylation-dependent fashion (Figure 6B and data not shown).

The SH2-SH3 adapter protein Nck associates with nephrin at Y1191 and $Y 1208$. Because we were interested initially in identifying protein-protein interactions that occur at the putative SH2 domainbinding motifs observed at Y1191 and Y1208, the putative interaction between nephrin and Nck was investigated in additional
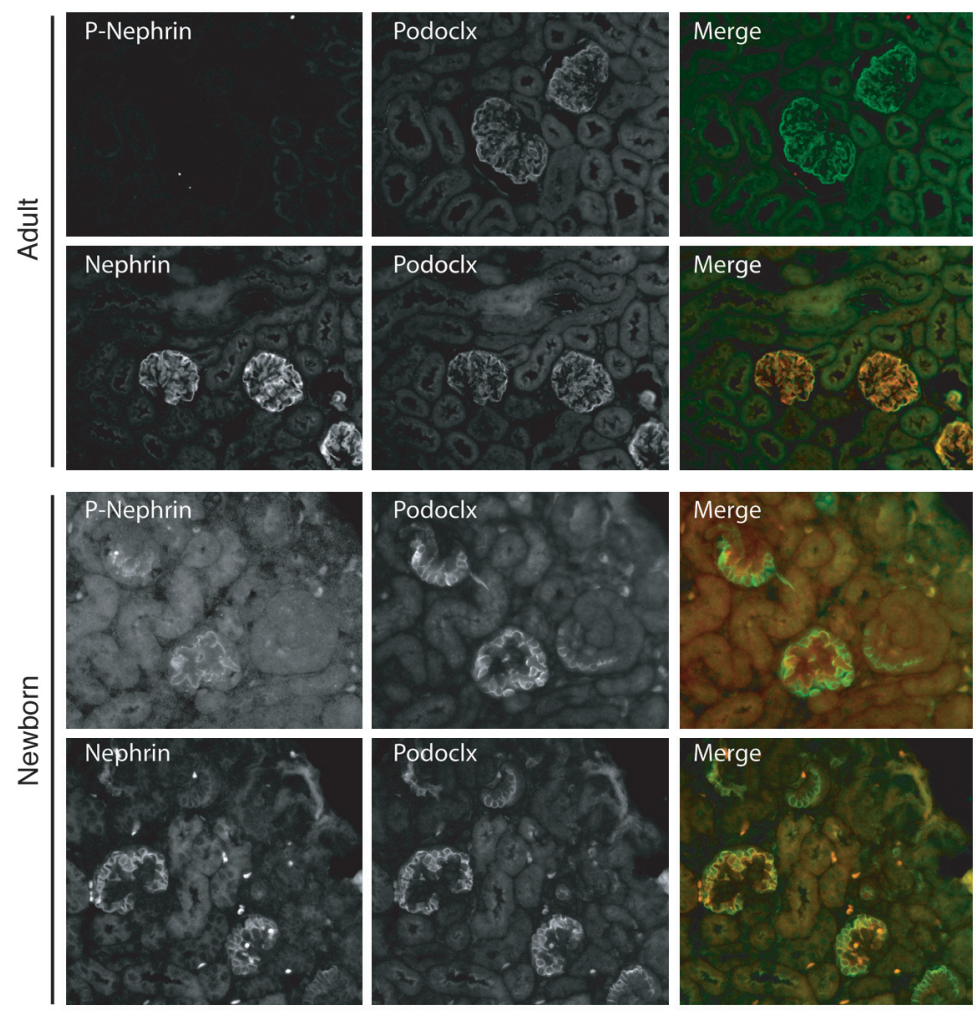

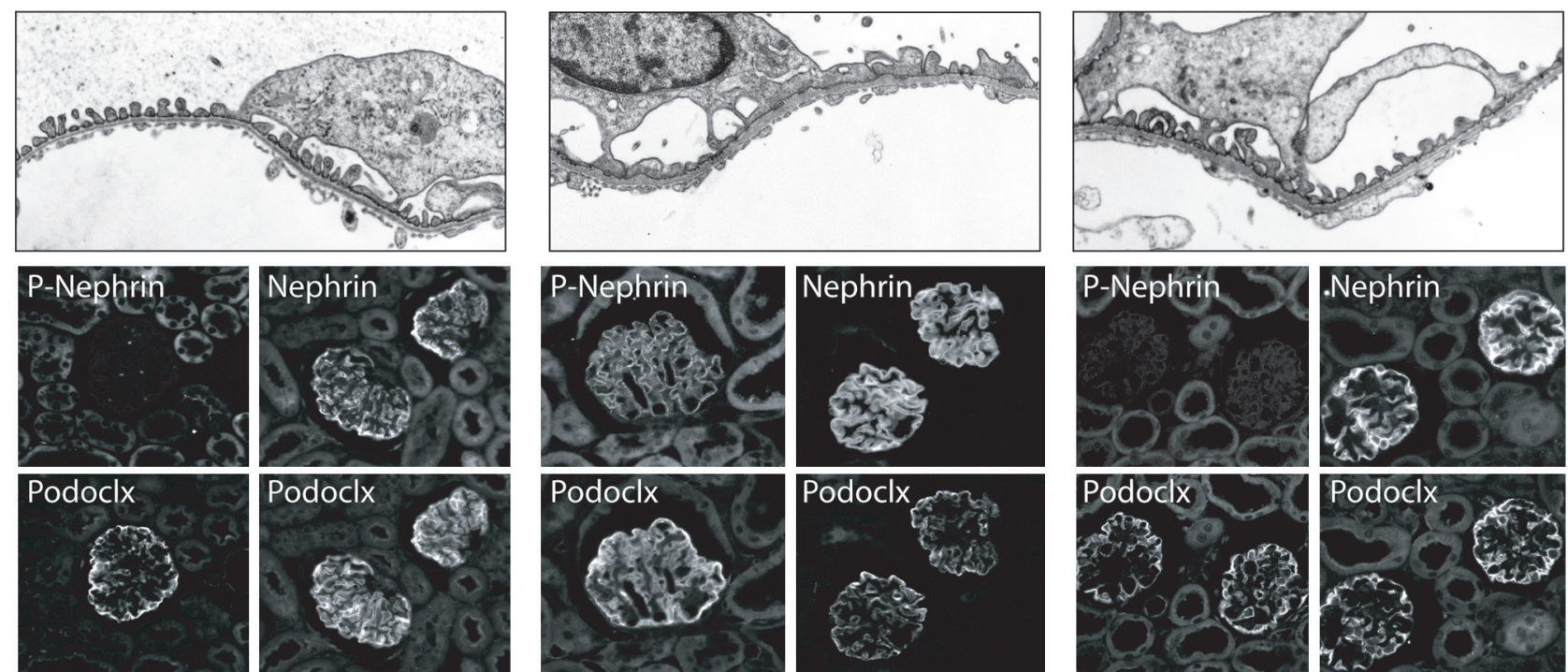
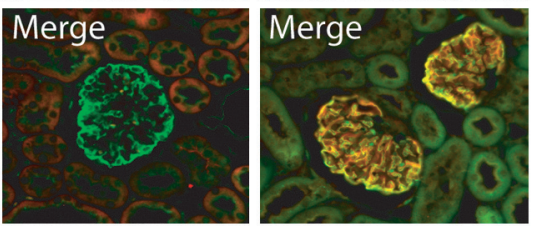

PBS (30 min)

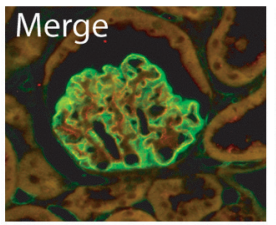

Protamine sulfate (15 min) PBS (15 min)
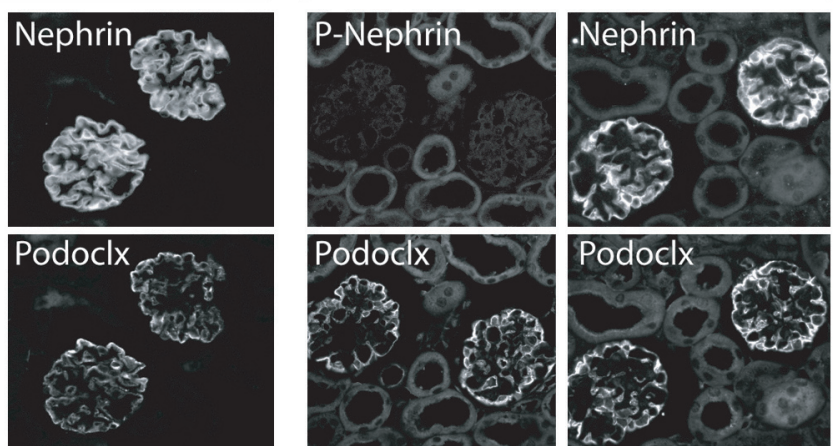

Podoclx
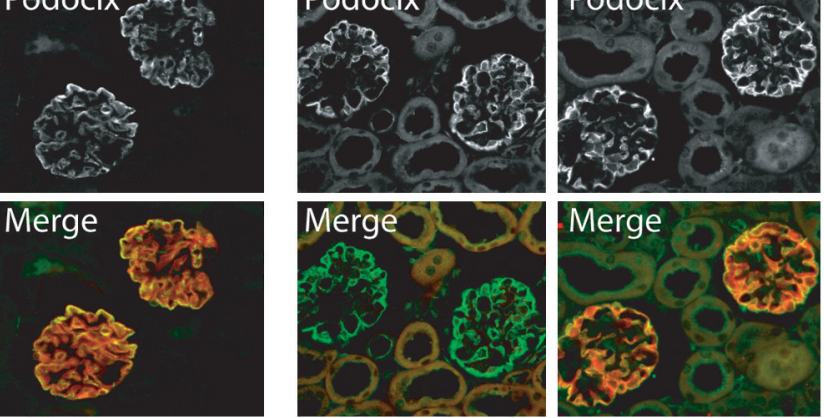

Protamine sulfate (15 min) Heparin sulfate (15 min)

\section{Figure 5}

Nephrin is transiently tyrosine phosphorylated during foot process effacement in the protamine sulfate-induced podocyte injury model. Adult mouse kidneys were perfused as indicated prior to perfusion fixation with paraformaldehyde. Sections were analyzed by electron microscopy (top row; magnification, $\times 2,800$ ) or by indirect immunofluorescence microscopy (bottom rows; magnification, $\times 400$ ) after incubating sections simultaneously with rabbit P-nephrin or total nephrin antibody and with mouse anti-podocalyxin antibody. Protamine sulfate rapidly induced foot process effacement; normal podocyte morphology was nearly restored following perfusion with heparin sulfate. P-nephrin immunoreactivity was detected following protamine sulfate perfusion, but detection was attenuated following perfusion with heparin sulfate.

detail. In mice, both Y1191 and Y1208 fall in the context of YDEV motifs that - relative to other SH2-binding domains - have been shown to have particularly high affinity for Nck (27). Moreover, as described in Discussion, the identification of a Nck-nephrin interaction would be consistent with our governing hypothesis that nephrin-dependent signaling - at least in part - serves to coordinate podocyte cytoskeletal dynamics since Nck is known to associate with proteins of the actin polymerization complex.

Initial experiments were directed at confirming that Nck is expressed in podocytes and that endogenous Nck associates with nephrin. Using Nck isoform-specific antibodies, both Nck1 and Nck2 were detected by immunoblotting in the lysates of a conditionally immortalized mouse podocyte cell line (28) cultured under either permissive or nonpermissive conditions (Figure 7A). Similarly, both Nck1 and Nck2 were detected in lysate obtained from isolated mouse glomeruli. Since nephrin is enriched in the podocyte DRM fraction, we examined whether Nck cofractionates with nephrin in DRM. Isolated mouse glomeruli were lysed at $4{ }^{\circ} \mathrm{C}$ in buffer containing $1 \%$ Triton $\mathrm{X}-100$, and the lysate was fractionated by flotation gradient centrifugation. Like nephrin and podocin, Nck2 (but not Nck1) was detected in the DRM fraction (Figure 7B).

A series of experiments was performed to confirm the association of nephrin and Nck and to map their interacting domains. To determine whether the nephrin-Nck association is mediated by a direct interaction, purified recombinant GST-nephrinCD expressed either in BL21 cells or in TKB1 cells was mixed with recombinant GST-Nck. Nck was immunoprecipitated from these mixtures, and after resolution by SDS-PAGE, immune complexes were examined by immunoblotting for nephrin or P-nephrin (Figure 8A). GST-nephrinCD associated with Nck by direct protein-protein interaction only when GST-nephrinCD was phosphorylated by expression in TKB1 cells. Nck did not associate with nephrin when Nck carried a previously described point mutation that disrupts the binding affinity of its $\mathrm{SH} 2$ domain (29). These results were confirmed in a mammalian (see Methods) cell system in which plasmids encoding nephrin and Nck with or without Fyn were transiently expressed (Figure 8B). As shown above (Figure 2D) in a similar experiment, transient transfection of plasmid encoding Fyn resulted in nephrin phosphorylation at Y1191 and Y1208. Here nephrin associated with Nck - but not Nck deleted of its SH2 domain (Nck $\Delta \mathrm{SH} 2)$ - only when Fyn was present. Together, these results are consistent with the conclusion that Nck directly interacts via its $\mathrm{SH} 2$ domain with a functional $\mathrm{SH} 2$-binding domain in nephrin when that domain is tyrosine phosphorylated. For reasons discussed above, it was anticipated that the functional nephrin SH2-binding domain(s) that interact with Nck are Y1191DEV and/or Y1208DEV. As shown in Figure 8C, tyrosine-phosphorylated wild-type nephrin, nephrin(Y1191F), 
A

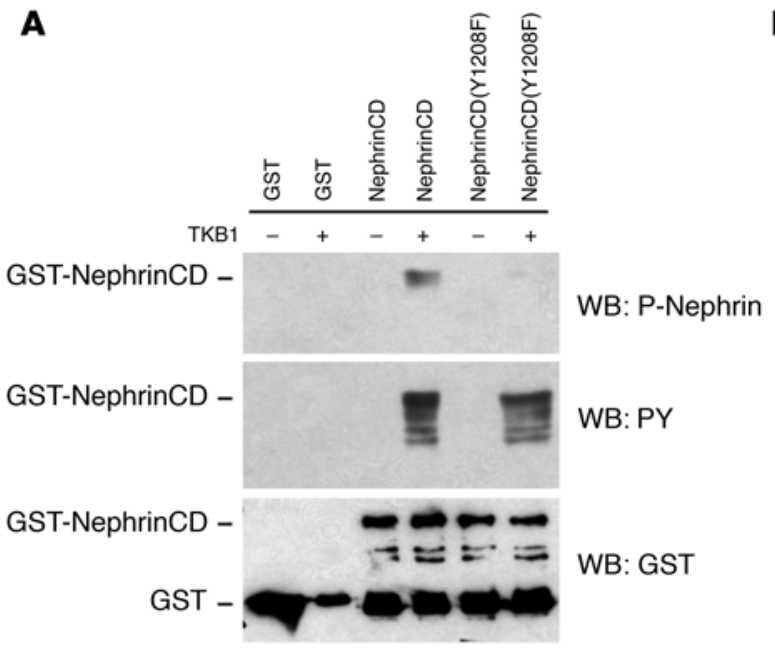

B

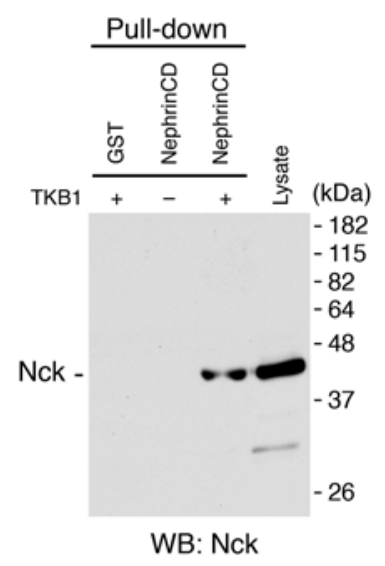

Figure 6

Nck associates with nephrin. GST-nephrinCD or its Y1208 mutant was expressed in BL21 or TKB1 E. coli and was affinity purified on glutathione agarose. (A) Immunoblotting with $\mathrm{P}$-nephrin or nonspecific PY antibody demonstrated that expression in TKB1 (but not BL21) cells resulted in phosphorylation on Y1208 and other nephrin tyrosine residues. (B) Purified recombinant GST alone or GST-nephrinCD obtained from either BL21 or TKB1 cells that was bound to glutathione agarose was incubated with isolated mouse glomerular lysate. Washed beads were eluted with glutathione. Eluate was resolved by SDS-PAGE and immunoblotted with the panel of antibodies shown in Table 2. Representative immunoblot was obtained using a pan-Nck antibody that demonstrated association of Nck with tyrosine-phosphorylated wild-type GST-nephrinCD but not unphosphorylated GST-nephrinCD or GST alone.

and nephrin(Y1208F) interacted with Nck, while nephrin mutated at both Y1191 and Y1208 did not.

Our previously published observations using Fyn-null mice showed that endogenous nephrin is a substrate of Fyn (23). Therefore, having demonstrated by employing in vitro techniques that nephrin and Nck interact in a SFK-dependent fashion, we sought to examine whether endogenous nephrin and Nck interact in a Fyndependent manner. In preliminary experiments, it was observed that isolation of glomeruli from mouse kidney by sieving induced nephrin phosphorylation on Y1191/1208 (data not shown). Using this experimental artifact to our advantage, the association of endogenous Nck and nephrin was examined. Isolated glomeruli obtained from wild-type mice were lysed in a buffer containing $1 \%$ NP-40 and $100 \mathrm{mM}$ potassium iodide. In reciprocal coimmunoprecipitation experiments employing this lysate, Nck associated with nephrin (Figure 8D). In contrast, association of nephrin and Nck was not demonstrable when immunoprecipitated complexes were obtained from glomeruli isolated by sieving from Fyn-null mice (Figure 8E).

Clustered nephrin CD becomes tyrosine phosphorylated, recruits Nck, and induces actin polymerization. Plasma membrane recruitment of Nck to several known tyrosine-phosphorylated transmembrane proteins results in concomitant recruitment of components of the actin polymerization complex and induction of localized actin polymerization $(30,31)$. We sought to test the hypothesis that in a similar fashion, recruitment of Nck to nephrin at the plasma membrane induces localized actin polymerization. Toward this end, a model fashioned after that described by Rivera et al. was employed (32). A series of fusion protein constructs was created in which the CD16 extracellular domain and the CD7 transmembrane domain was coupled to either nephrinCD or control cytoplasmic domains that included the HA epitope tag and GFP (CD16/7/

nephrinCD, CD16/7/HA, and CD16/7/ GFP; Figure 9A); these fusion proteins were expressed in NIH $3 \mathrm{~T} 3$ cells by transient transfection. After 30 hours, mouse anti-CD16 antibody and a secondary antimouse IgG antibody were added to the culture media of live cells. This maneuver induced visible aggregation, or clustering, of CD16/7 fusion proteins on the plasma membrane (Figure 9B and Figures 10 and 11) and induced tyrosine phosphorylation on Y1191/1208 of CD16/7/nephrinCD (Figure 9C). Aggregation-induced tyrosine phosphorylation on these sites occurred within 1 minute of addition of aggregating antibodies and was dependent on SFK activity, since induction of tyrosine phosphorylation was inhibited in the presence of PP2. The observation that with aggregation CD16/7/nephrinCD became phosphorylated on Y1191/1208 predicted that Nck would be recruited to CD16/7/ nephrinCD at the plasma membrane. To test this prediction, NIH 3T3 cells were cotransfected with plasmid encoding CD16/7/nephrinCD and Nck-GFP and were examined by immunofluorescence confocal microscopy. In the presence of aggregating antibody, Nck-GFP colocalized in clusters with CD16/7/nephrinCD at the plasma membrane (Figure 10). We did not observe a similar pattern of Nck-GFP clus-

\section{Table 2}

Antibodies to SH2-binding domain-containing proteins used in this experiment

SH2 domain protein
Adaptors
C-Cbl
Crk
Grb2
Nck
PLC $\gamma$
PI3K
PTP1C/SHP1
PTP1D/SHP2
Ras-GAP
ShcA
Tensin
Vav
Protein kinases
CSK
C-Src
Fyn
Lyn
JAK1
Ntk
Tyk2
Tek
Itk

Interaction detected 


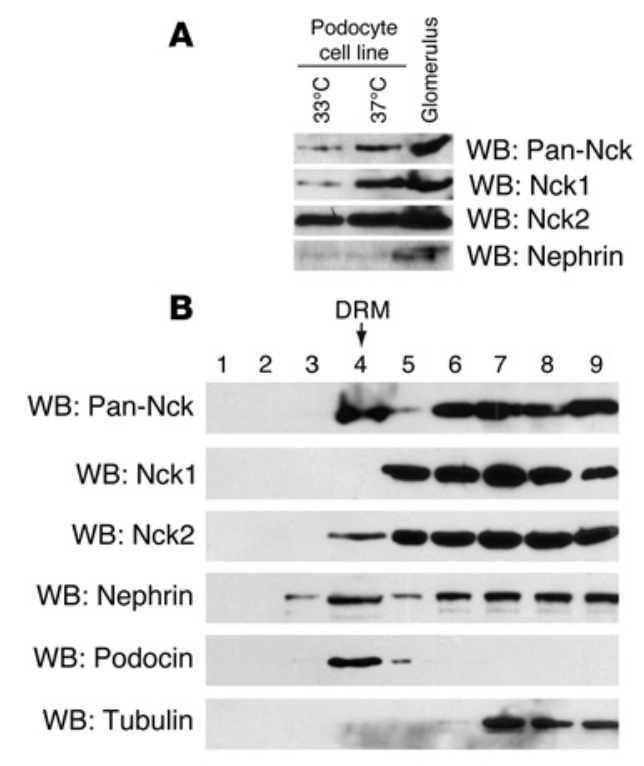

tering at the plasma membrane when nephrinCD was substituted with an HA epitope tag or when $\mathrm{Nck} \Delta \mathrm{SH} 2$ was substituted in similar experiments. When aggregating antibody was not added to cells coexpressing CD16/7/nephrinCD and Nck-GFP, aggregation of $\mathrm{Nck}$ at the plasma membrane was not detected. In summary, these results suggest that clustering of nephrinCD results in nephrin tyrosine phosphorylation and recruitment of Nck to nephrin in an Nck-SH2 domain-dependent fashion.

We also tested whether aggregation of CD16/7/nephrinCD was sufficient to induce localized actin polymerization in this model. Live cells expressing CD16/7/nephrinCD, Nck-cyan fluorescent protein (Nck-CFP), and actin-GFP were treated by adding aggregating antibodies to culture media; cells were then fixed and evaluated by fluorescence confocal microscopy (Figure 11). Under these conditions, the actin cytoskeleton was dramatically altered. Discreet actin pedestals were observed as large, intensely GFP-positive foci. Invariably, these actin pedestals colocalized with CD16/7/nephrinCD clusters at the apical plasma membrane. Also noted frequently were actin tails, representing actin filaments that extended from the apical plasma membrane at sites of clustered CD16/7/nephrinCD (Figure 11, insets). In control experiments, induction of actin polymerization at the plasma membrane was not observed when clustering antibodies were not present. Moreover, clustering of CD16/7/HA failed to induce detectable localized actin polymerization. Together these results support the conclusion that clustering of nephrin in this model is sufficient to recruit Nck to the plasma membrane and to induce actin polymerization. In a final experiment, $\mathrm{Nck} \Delta \mathrm{SH} 2$ was coexpressed with CD16/7/nephrinCD and actin-GFP to investigate whether Nck was necessary as an intermediate in clustered CD16/7/nephrinCD-induced actin polymerization. Remarkably, under these conditions, clustering of CD16/7/nephrinCD failed to induce actin polymerization (Figure 11).

\section{Discussion}

The nephrin protein complex appears to play an important role in specifying cell junction formation by promoting interactions between specific cell types and in doing so participates in defin-

\section{Figure 7}

Nck1 and Nck2 are present in podocytes. (A) Lysate obtained from an immortalized podocyte cell line cultured under permissive $\left(33^{\circ} \mathrm{C}\right)$ or nonpermissive $\left(37^{\circ} \mathrm{C}\right)$ conditions or obtained from isolated mouse glomeruli was resolved by SDS-PAGE and immunoblotted using the indicated antibodies. (B) Mouse glomeruli isolated by sieving were extracted in buffer containing $1 \%$ Triton $\mathrm{X}-100$ at $4^{\circ} \mathrm{C}$. The lysate was subjected to flotation gradient centrifugation. DRM was identified in lane 5 at the interface between 5 and 30\% Optiprep densities. Fractions $(1 \mathrm{ml}$ ) were collected and analyzed by immunoblotting with the indicated antibodies.

ing tissue organization. In C. elegans, nephrin and Neph1 homo$\log$ ligation is necessary for directing synapse formation between a specific neuron and its specific target epithelial tissue $(9,11)$. Similarly, in D. melanogaster, nephrin-Neph1 homolog interaction influences compound eye morphogenesis and patterning by specifying junction formation between appropriate cell types (10). In a potentially related fashion in the podocyte, nephrin is necessary for establishing normal podocyte foot process morphology and normal intercellular junction structure $(3,5,15,23,33,34)$.

In general, the dynamic process of epithelial cell junction formation is dependent on proper regulation and integration of junctional adhesion with actin cytoskeletal dynamics (35). This is a complex process, the details of which likely vary according to cell type. Nevertheless, central to this process is precisely regulated actin polymerization and organization; actin polymerization appears to provide the motive force necessary for junction formation, and disruption of the molecular machinery necessary for regulating actin cytoskeletal dynamics prevents proper junction formation (36). Therefore the nephrin family of transmembrane proteins might function in specifying junction formation in part by transducing signals that serve to regulate associated cytoskeletal dynamics proximal to the forming junction.

The observation that mutation or deletion of nephrin results in both failure of proper foot process morphogenesis and concomitant proteinuria first suggested the hypothesis that nephrin serves as a component of a signaling complex that directly integrates podocyte junctional integrity with cytoskeletal dynamics $(3,4,6$, 14). The observations made herein provide the first direct evidence to our knowledge for a tyrosine phosphorylation-mediated signaling mechanism by which this integrative function is derived. These data indicate that engagement of the nephrin ectodomain by its ligand induces SFK activity resulting in tyrosine phosphorylation of the nephrinCD on specific tyrosine residues. Subsequently, the adapter protein Nck and presumably proteins associated with Nck are recruited to phosphorylated nephrin, resulting in Nck-dependent regulation of local actin dynamics.

The discovery that Nck associated with activated nephrin and was necessary for nephrin-directed actin polymerization meshes well with the hypothesis that nephrin is one element in a mechanism that serves to integrate podocyte intercellular junction structure with cytoskeletal dynamics. As demonstrated in several systems, Nck serves as an adaptor protein that recruits to a plasma membrane locus a critical concentration of N-WASp, components of the Arp2/3 complex, and other components of the actin polymerization machinery sufficient to induce and regulate actin polymerization (30-32, 37-39). Similar mechanisms have been identified in a number of mammalian receptor systems including the EGF, PDGF, and T cell receptor complexes where 


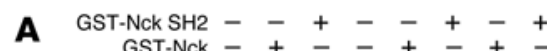
GST-NCk $-+{ }_{-}^{+}+-+$

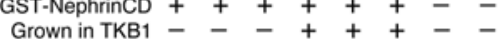

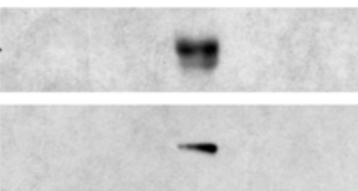

IP: Nck

WB: P-Nephrin

IP: Nck

WB: Nephrin
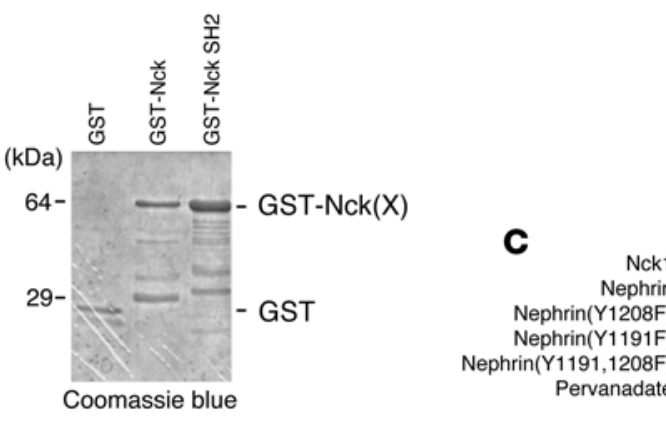

B

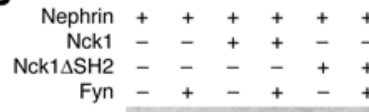

Fyn

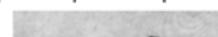

IP: Nephrin WB: Nck

$-\infty-\infty$ WB: Nephrin

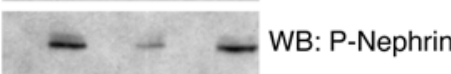

WB: Nck

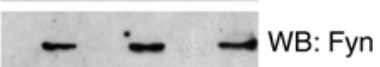

$\begin{array}{llllllllll}\text { ck1 } & + & + & + & + & + & + & + & + & + \\ \text { hrin } & + & + & - & - & - & - & - & - & - \\ 8 F) & - & - & + & + & - & - & - & - & - \\ 1 F) & - & - & - & - & + & + & - & - & - \\ 8 F) & - & - & - & - & - & - & + & + & - \\ \text { date } & - & + & - & + & - & + & - & + & -\end{array}$

IP: Nephrin

WB: Nck

WB: Nephrin(X)

D

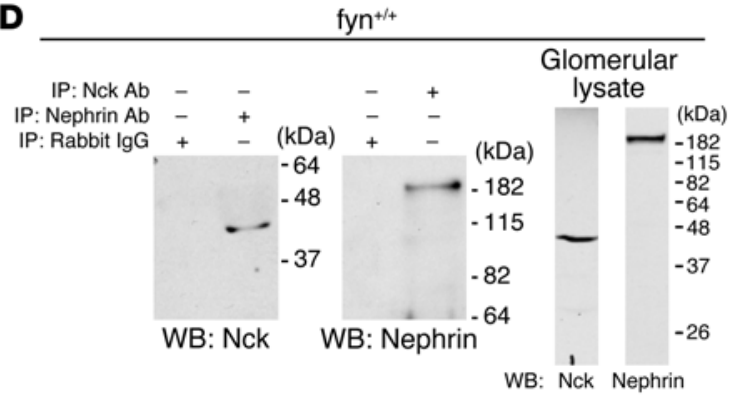

E

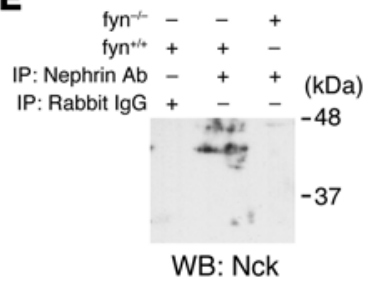

\section{Figure 8}

Nck binds directly with nephrin via an SH2 domain-dependent interaction. (A) Purified recombinant GST-nephrinCD was prepared from BL21 cells or TKB1 cells and was incubated in vitro with GST-Nck or a Nck mutant in the SH2 domain. The specified mixtures of recombinant proteins were immunoprecipitated with anti-Nck. Immune complexes were resolved by SDS-PAGE and were immunoblotted with either nephrin or P-nephrin antibody. Coomassie blue-stained gel demonstrates GST-Nck proteins used in this experiment. (B) COS7 cells were transiently transfected as indicated with plasmids encoding nephrin and Fyn. Independently, COS7 cells were transfected with plasmid encoding Nck1 or Nck1 $1 \mathrm{SH} 2$. Lysate obtained from these independently transfected cells were mixed, then immunoprecipitated and/or immunoblotted as indicated. (C) COS7 cells were transfected with plasmid encoding nephrin or its tyrosine mutants, then treated with pervanadate for 15 minutes as indicated prior to lysis. Independently, COS7 cells were transfected with plasmid encoding Nck1. Lysate obtained from these independently transfected cells were mixed, then immunoprecipitated and/or immunoblotted as indicated. (D) Coimmunoprecipitation experiments from isolated wild-type mouse glomerular lysate using pan-Nck and nephrin antibodies demonstrating association of endogenous Nck and nephrin. (E) Similar experiments performed on isolated glomerular lysate obtained from wild-type and Fyn-null mice.

Nck has been implicated as necessary in actin dynamics-dependent processes $(30,31,37)$. A related mechanism is employed by several bacterial and viral pathogens $(38,39)$. For example, insertion of the enteropathogenic E. coli proteins Tir and intimin into the plasma membrane of host epithelial cells triggers robust actin pedestal formation at the plasma membrane when Tir is tyrosine phosphorylated by Fyn, creating a docking site for host cell-derived Nck and associated N-WASp (38). The nephrin receptor complex appears to employ a remarkably similar mechanism for coordinating junctional events with podocyte cytoskeletal dynamics. Like other systems, nephrin at the podocyte intercellular junction appears to recruit a high local concentration of Nck, which facilitates actin polymerization because it provides multiple docking sites for Nck and because nephrin itself is concentrated by clustering in a lipid microdomain (32).

Nephrin protein is first expressed when podocyte process formation is initiated in the podocyte precursor (7). During this event, processes emerge from the basolateral aspect of the precursor cell in a polarized fashion and appear to push into neighboring cells; here nephrin is targeted specifically to the newly forming intercellular junctions (7). Phosphorylation on mouse nephrin Y1191 and/ or Y1208 occurred transiently during glomerulogenesis during this process of podocyte intercellular junction formation. Considered in the context of results obtained in cell culture, it is reasonable to hypothesize that within the glomerulus, nephrin is tyrosine phosphorylated upon ectodomain engagement during this event. 


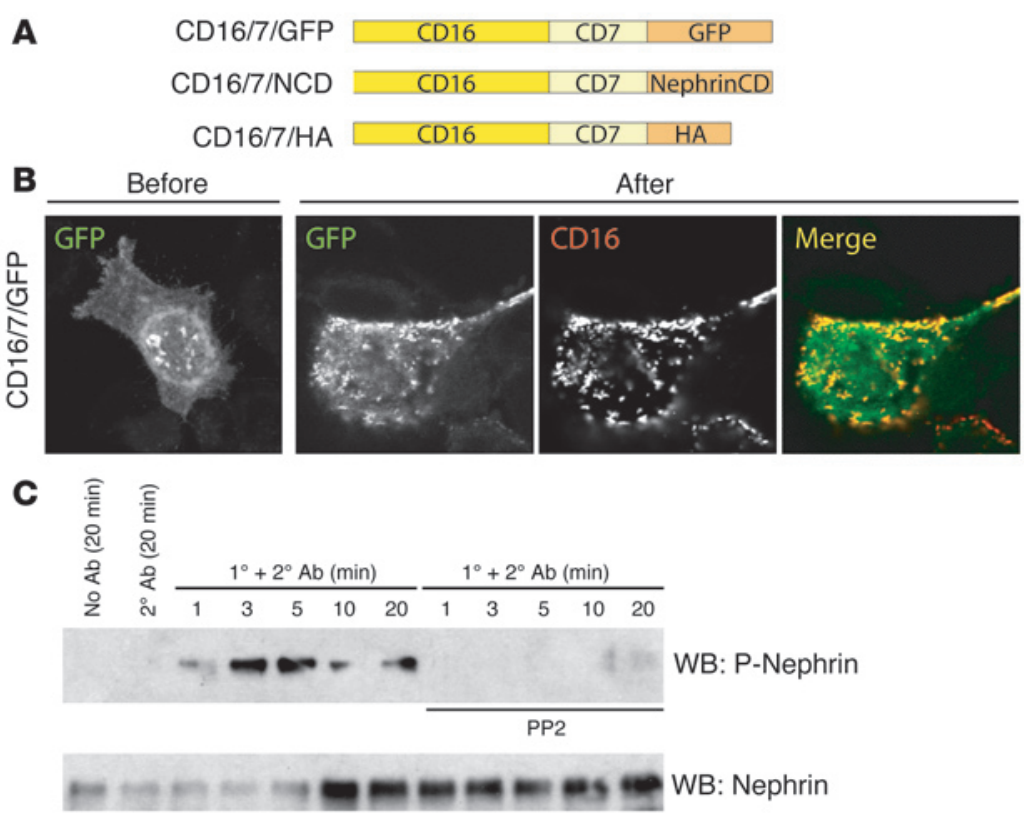

\section{Figure 9}

Clustering of CD16 chimeric proteins and induction of tyrosine phosphorylation on clustered CD16/7/nephrinCD (CD16/7/NCD). (A) Schematic representation of CD16 fusion proteins prepared for these experiments. (B) Confocal microscopy imaging of NIH 3T3 cells expressing the CD16/7/GFP fusion protein before and after addition to media of live cells of anti-CD16 antibody and rhodamine-conjugated anti-IgG antibody. In the merged image at right, rhodamine IgG (red) and GFP (green) colocalized (yellow) on the plasma membrane, demonstrating clustering of the CD16/7/GFP chimeric protein. Note absence of clusters where antiCD16 antibody was not present. Magnification, $\times 600$. (C) NIH 3T3 cells expressing CD16/7/nephrinCD were treated with clustering antibodies $\left(1^{\circ}+2^{\circ} \mathrm{Ab}\right)$ or as indicated for the time periods shown, then immunoblotted with P-nephrin antibody to detect phosphorylation of Y1191 and Y1208. Pretreatment of cells with PP2 blocked tyrosine phosphorylation on these sites following induction of clusters.
Subsequent Nck recruitment to phosphorylated nephrin likely initiates directed actin polymerization and/or elongation at this site and by participating in regulating actin dynamics provides one element in a mechanism that specifies the specialized structure of the podocyte foot process and intercellular junction. Consistent with a role of Fyn in initiating this process is the observation that genetic deletion of Fyn in mice results in attenuated nephrin phosphorylation and is associated with abnormal foot process morphology (7, 23). The observation that in situ deletion of Fyn in the podocyte precluded recruitment of Nck to nephrin strengthens the conclusion that this pathway is operative in nature.

Phosphorylation of nephrin Y1191/1208 during glomerulogenesis is transient since nephrin does not remain phosphorylated on these sites in the fully differentiated adult glomerulus. What does the transient nature of phosphorylation on these residues suggest about the function of Nck in this system? While investigation of this process is required, it is possible that tyrosine phosphorylation-dependent Nck recruitment might be necessary only during active actin polymerization or elongation that occurs during junction formation. Like similar junctional receptor complexes, it is clear that actin filaments can associate with the slit diaphragm junctional complex via multiple intermediaries (e.g., at the podocyte intercellular junction via CD2ap, ZO-1/Neph1, JAM, etc.) that might provide stable, phosphorylation-independent contacts necessary for maintaining the structural integrity of foot processes and their formed intercellular junctions. In recent work the classical dogma that actin binds stably to classical cadherins at mature epithelial adherens junctions was challenged; it was suggested that cadherin-catenin complexes play a dynamic regulatory role, participating only in weak interactions with the cytoskeleton (40, 41). In a similar fashion, nephrin might play a dynamic regulatory role without necessarily forming stable interactions with the actin cytoskeleton via Nck.

It has been well appreciated that in human glomerular disease proteinuria is invariably associated with foot process effacement. Presumably, in the setting of disease, these integrated processes occur following podocyte injury that results in an inside-out signaling event culminating in effacement and alteration of the intercellular junction. Nephrin phosphorylation also occurred rapidly following induction of foot process effacement in the protamine sulfate model. At first inspection, this observation appears to contradict the model proposed above that Y1191/1208 phosphorylation initiates actin dynamics necessary for junction formation. Indeed, one might argue that because phosphorylation on Y1191/1208 is temporally associated with effacement that this phosphorylation event results in disassembly of the podocyte intercellular junction. However, deletion of Fyn in mouse podocytes was not associated with stabilization of the podocyte intercellular junction; rather, it was associated with failure of appropriate junction formation. Moreover, it is difficult to overlook the established biology of Nck recruitment in other systems in which Nck participates in junction formation. Therefore, while additional investigation is necessary to confirm the hypothesis, it is possible that Y1191/1208 phosphorylation occurs after podocyte injury as the podocyte actin cytoskeleton reorganizes; this might require reinduction of actin polymerization as new, albeit different, podocyte intercellular junctions are created.

Using both biochemical and genetic approaches, previous work demonstrated that Fyn can directly bind and phosphorylate nephrin in the context of the podocyte lipid raft $(22,23,42)$. The precise mechanism by which nephrin phosphorylation is initiated remains unresolved. In similar lipid raft-based receptor systems, it has been suggested that ectodomain engagement results in either (a) recruitment of the membrane-associated receptor to the lipid raft, where it is brought into proximity of the resident Src kinase and is tyrosine phosphorylated; or (b) clustering of unique rafts such that raft components are combined, leading to initiation of cellular signaling (reviewed in ref. 24). One or both of these general mechanisms may hold for nephrin since it has been shown that podocin serves as a slit diaphragm lipid raft scaffold that recruits nephrin to the lipid raft $(20,43)$. However, at least in the artificial HEK 293 cell system, physical clustering of nephrin in a lipid raft does not appear to be required for induction of SFK catalytic activity and nephrin phosphorylation, 


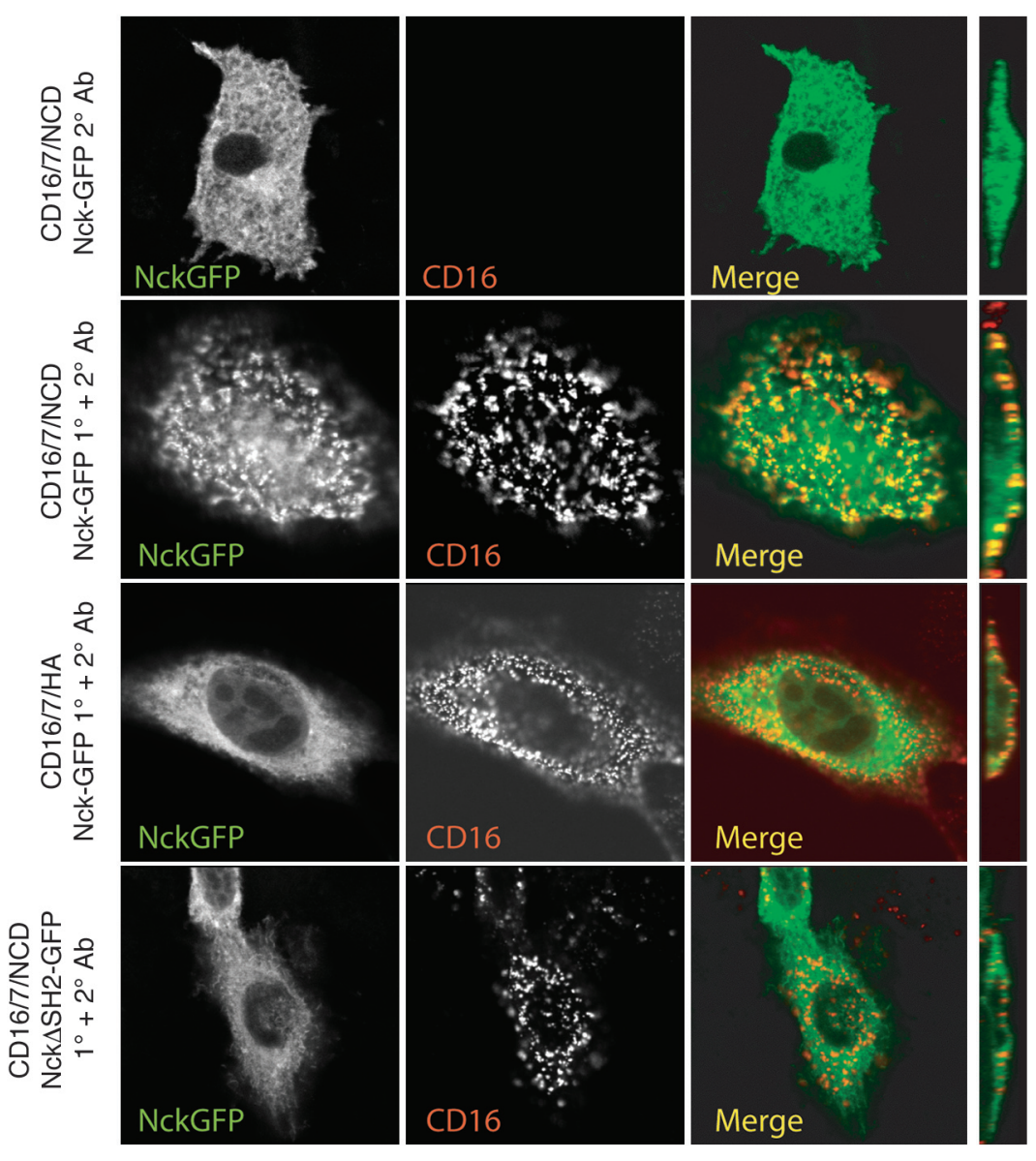

\section{Figure 10}

Nck is recruited to CD16/7/nephrinCD clusters at the plasma membrane. NIH 3T3 cells expressing indicated chimeric proteins (red) and Nck-GFP or Nck $\Delta$ SH2GFP (green) were treated with anti-CD16 antibody and rhodamine anti-lgG antibody or were treated with the secondary antibody only, then fixed and examined by confocal microscopy. Far right panels are images reconstructed in the $Y Z$ plane. Colocalization of Nck-GFP and CD16/7/nephrinCD appear yellow on merged images. Data are representative of multiple experiments. Magnification, $\times 600$.

raised against a HPLC-purified synthetic oligopeptide WGPLPYDEVQM manufactured at the Protein Core of the University of Michigan Medical School. Obtained antiserum was affinity purified commercially by Fabgennix Inc. using the phosphorylated peptide coupled to sepharose beads as described by the manufacturer. This antibody was characterized as described herein.

Plasmids. Plasmid encoding GST-nephrinCD fusion protein, which includes the entire cytoplasmic domain of mouse nephrin was prepared in PGEX4T-1 (Amersham Biosciences) using standard techniques (23). Plasmids encoding the various GST-nephrinCD tyrosine residue point mutants were prepared by standard PCR cloning techniques using GST-nephrinCD plasmid as a template (48). GST-Nck1 and GST-Nck1-SH2 mutants were previously described (29). Recombinant GST fusion proteins were prepared and purified from bacterial lysates as described previously (48). GST-nephrinCD or its mutants were expressed in and purified from TKB1 cells (Strategene). Mammalian plasmids encod-

since addition of the 50A9 monoclonal antibody alone, without a secondary clustering antibody, induced both events. Therefore engagement of the nephrin ectodomain might induce a nephrin conformational change that by a yet-to-be-identified mechanism transduces an SFK-activating signal.

Nephrin is necessary for establishing selective glomerular filtration. In part, this function may be dependent on the 3D structure created by the cis-and trans-junctional interactions of nephrin with other transmembrane components at this site $(44,45)$. However, given the generally accepted function of coordinated cytoskeletal dynamics in establishing intercellular junctional architecture (36), the role of nephrin in regulating cytoskeletal dynamics may be as important in establishing a filtration barrier as nephrin's potential for directly creating a physical barrier to the passage of macromolecules at the junction.

\section{Methods}

Antibodies. Rabbit polyclonal antisera against mouse nephrin (7); mouse podocin (44); and pan-Nck, Nck1, and Nck2 (46) were described previously. Chicken polyclonal anti-podocalyxin was a gift of D. Kershaw (University of Michigan; ref. 47). Commercially distributed antibodies employed included antibodies against Grb2, Fyn, glutathione S-transferase, Grb2, PTP1C, PTP1D, Ras-GAP, c-Src, Lyn, and JAK1 (Santa Cruz Biotechnology Inc.); Src(Y418) (BioSource International); Yes, c-Cbl, PI3K, tensin, Vav, Csk, Itk, and Tek (Upstate USA Inc.); P-tyrosine and $\alpha$-tubulin (Sigma-Aldrich); Crk (BD Biosciences - Pharmingen); PLC $\gamma$ (BD Biosciences) and ShcA (BD). A rabbit polyclonal phosphospecific antibody was ing mouse nephrin (7), Fyn (49), human nephrin (gift of K. Tryggvason, Karolinska Institute, Stockholm, Sweden; ref. 21), and Flag-tagged Nck1 and Nck1 $\triangle \mathrm{SH} 2$ (50) were previously described. Mammalian expression plasmids encoding mouse nephrin point mutants Y1191F, Y1208F, and Y1191/1208F were prepared in pcDNA3.1 (Invitrogen Corp.) using standard PCR methods. Restriction digestion and DNA sequencing were used to validate all constructs.

Metabolic orthophosphate labeling of isolated glomerular DRM. Glomerular DRM fractions were isolated, resuspended in kinase buffer $(25 \mathrm{mM}$ HEPES, pH 7.2, $10 \%$ glycerol, $100 \mathrm{mM} \mathrm{NaCl}, 5 \mathrm{mM} \mathrm{MgCl}_{2}, 5 \mathrm{mM} \mathrm{MnCl}_{2}$, $0.1 \mathrm{mM}$ sodium orthovanadate, and a mixture of protease inhibitors) and pelleted by centrifugation at $16,000 \mathrm{~g}$ at $4^{\circ} \mathrm{C}$. These pellets were resuspended in kinase buffer containing $25 \mu \mathrm{M}$ ATP and $5 \mu \mathrm{Ci}\left[{ }^{32} \mathrm{P}-\gamma\right] \mathrm{ATP}(3,000$ $\mathrm{Ci} / \mathrm{mmol}$ ) and incubated at $25^{\circ} \mathrm{C}$ for 15 minutes. Pellets were pelleted and washed once with kinase buffer lacking ATP $\mathrm{MgCl}_{2}$ and $\mathrm{MnCl}_{2}$. Pellets were extracted with RIPA buffer and centrifuged at $16,000 \mathrm{~g}$, and the obtained supernatants were retained for additional processing.

In vitro phosphate labeling. GST-nephrinCD $(1 \mu \mathrm{g})$ was incubated with $1 \mu \mathrm{g}$ recombinant Fyn (Upstate USA Inc.) in kinase buffer containing $25 \mu \mathrm{M}$ ATP and $5 \mu \mathrm{Ci}\left[{ }^{32} \mathrm{P}-\gamma\right] \mathrm{ATP}(3,000 \mathrm{Ci} / \mathrm{mmol})$ and incubated at $25^{\circ} \mathrm{C}$ for 15 minutes. Reactions were terminated with SDS loading buffer and boiled for 5 minutes. Samples were separated by reducing SDS-PAGE and transferred to nitrocellulose, and phosphorylated nephrin was detected by autoradiography. The radioactive band was excised and further processed for phosphopeptide mapping.

Phosphopeptide mapping. Tryptic phosphopeptide 2D mapping was performed as described by Boyle et al. (51). Following phosphorylation of 

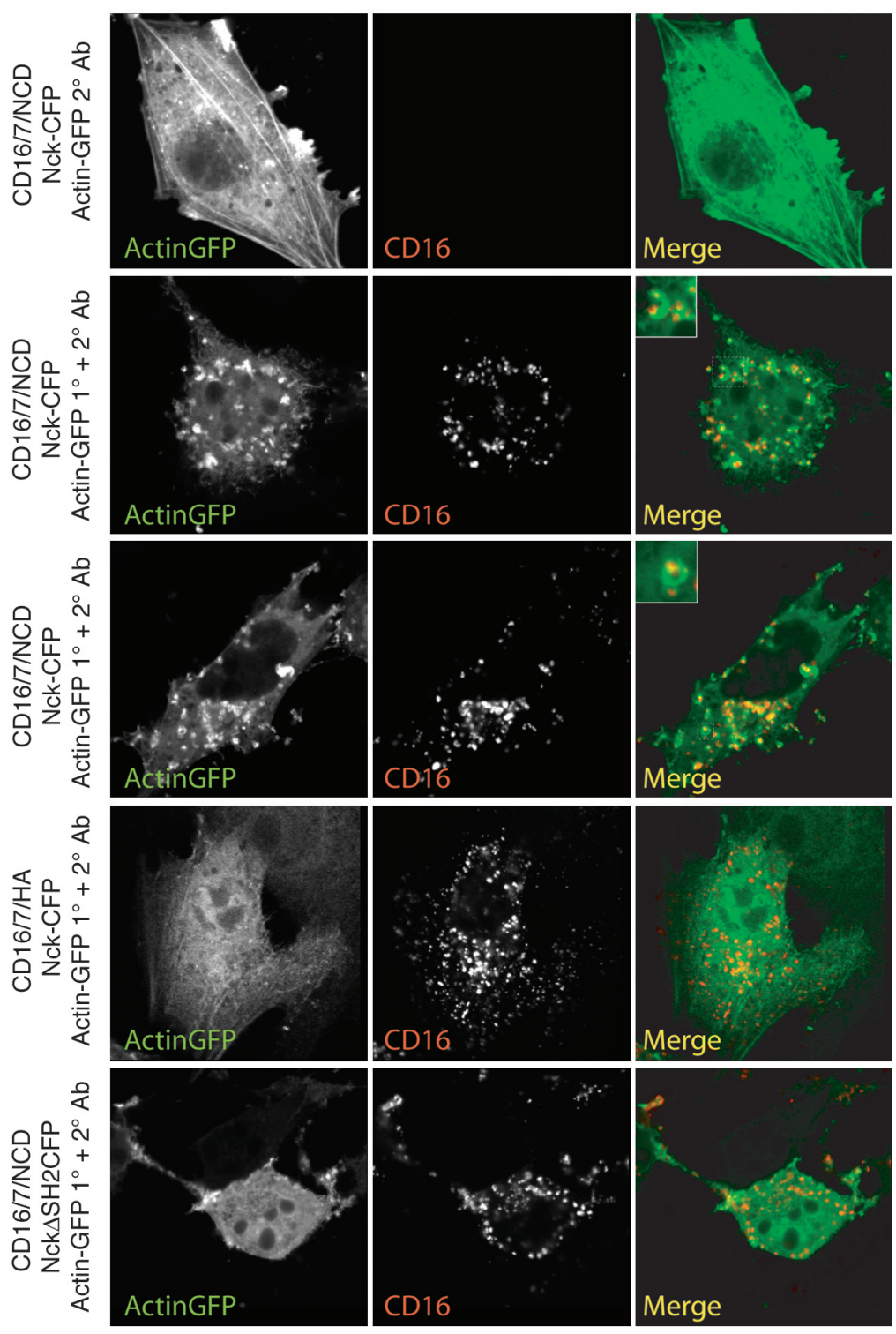

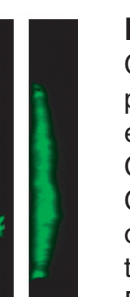

Figure 11

Clustered nephrinCD induces Nck-dependent actin polymerization. NIH 3T3 cells expressing the indicated chimeric proteins (red), Nck-CFP or Nck $\Delta \mathrm{SH} 2-$ CFP, and actin-GFP (green) were treated with antiCD16 antibody and rhodamine anti-IgG antibody or were treated with the secondary antibody only, then fixed and examined by confocal microscopy. Far right panels are images reconstructed in the $Y Z$ plane. Two representative examples of changes that occur in the presence of actin-GFP, Nck-CFP, and CD16/7/nephrinCD are shown in the second and third rows - note the intense green actin-GFP signal emitted by multiple actin tails or pedestals was associated nearly invariably with the red signal that marked clustered CD16/7/nephrinCD at the plasma membrane (insets). Data are representative of multiple experiments. Magnification, $\times 600$; $\times 1,200$ (insets).

Cell culture. Transient transfections were carried out in COS7 or SYF cells that were cultured in DMEM supplemented with 10\% FCS (Invitrogen Corp.) and $200 \mathrm{U} / \mathrm{ml}$ penicillin and streptomycin (Roche Applied Science). Transfections were performed using Fugene- 6 (Roche Applied Science) according to the manufacturer's directions. Cells were treated with $50 \mu \mathrm{M}$ pervanadate for 20 minutes prior to harvesting cells. HEK 293 cells and their derivatives were grown in Eagle's minimal essential medium with Earle's BSS supplemented with $2 \mathrm{mM}$ L-glutamine, $1.0 \mathrm{mM}$ sodium pyruvate, $0.1 \mathrm{mM}$ nonessential amino acids, $1.5 \mathrm{~g} / \mathrm{l}$ sodium bicarbonate, $10 \%$ fetal bovine serum, $100 \mathrm{U} / \mathrm{ml}$ penicillin, and $100 \mu \mathrm{g} / \mathrm{ml}$ streptomycin. HEK 293 cells stably expressing human nephrin or the Y1191F, Y1208F, or Y1191/1208F point mutants of nephrin were generated by transfecting HEK 293 cells with corresponding plasmids; following selection in G418 $(1 \mathrm{mg} / \mathrm{ml})$, cells were pooled and maintained in selection media. Conditionally immortalized mouse podocytes (gift of K. Endlich, University of Heidelberg, Heidelberg, Germany; ref. 28) were cultured under permissive

nephrin in vivo or phosphorylation of GST-nephrinCD or its mutants in vitro, proteins were immunoprecipitated, resolved by SDS-PAGE, and transferred to a nitrocellulose membrane. The nephrin band was identified by autoradiography and excised, and the membrane was soaked in $0.5 \%$ polyvinylpyrrolidone (PVP-360) in $100 \mathrm{mM}$ acetic acid for $30 \mathrm{~min}$ utes at $37^{\circ} \mathrm{C}$, then washed extensively with water and $0.05 \mathrm{M} \mathrm{NH}_{4} \mathrm{HCO}_{3}$. Samples were digested in sequencing grade trypsin $(20 \mu \mathrm{g}$ in $200 \mu \mathrm{l}$ of $50 \mathrm{mM} \mathrm{NH}_{4} \mathrm{HCO}_{3}$ overnight at $37^{\circ} \mathrm{C}$; Pierce Biotechnology). Resulting supernatants were dried, and samples were resolubilized in $\mathrm{pH} 1.9$ buffer containing $2.5 \%$ (vol/vol) formic acid (88\%) and 7.8\% (vol/vol) acetic acid. Aliquots of each sample containing 20,000 cpm were spotted onto $20 \times 20$ $\mathrm{cm}$ thin-layer cellulose plates and separated in the first dimension by electrophoresis for 45 minutes at $1,000 \mathrm{~V}$ using buffer containing $2.5 \%$ formic acid and 7.8\% acetic acid at $\mathrm{pH}$ 1.9. After drying, peptides were further separated in the second dimension by ascending chromatography for 8 hours using buffer containing 37.5\% (vol/vol) 1-butanol, 25\% (vol/vol) pyridine, and $5 \%$ (vol/vol) acetic acid. The pattern of tryptic phosphopeptides was visualized by exposing the dried cellulose plates to a PhosphoImager plate (model Storm-860; Amersham Biosciences). conditions at $33^{\circ} \mathrm{C}$ in DMEM containing $10 \% \mathrm{FCS}, 10 \mathrm{U} / \mathrm{ml}$ interferon- $\gamma$ (Roche Diagnostics Corp.), penicillin, and streptomycin. These cells were switched to nonpermissive conditions by culturing in same media deficient of interferon at $37^{\circ} \mathrm{C}$. NIH $3 \mathrm{~T} 3$ cells were cultured on prepared cover slips in DMEM supplemented with $10 \%$ fetal bovine serum, $100 \mathrm{U} / \mathrm{ml}$ penicillin, and $100 \mu \mathrm{g} / \mathrm{ml}$ streptomycin. Cover slips were treated with $1 \mathrm{M} \mathrm{HCl}$ at $50-60^{\circ} \mathrm{C}$ for 16 hours, cooled, and washed extensively in distilled and double-distilled $\mathrm{H}_{2} \mathrm{O}$. Cover slips were washed in ethanol and left to dry between folded sheets of filter paper. Before use, cover slips were treated with $100 \%$ ethanol in an open tissue culture disc located in a fume hood and allowed to dry. Cover slips were added to individual wells and coated with fibronectin (Chemicon International) at a concentration of $0.1 \mu \mathrm{g} / \mathrm{ml}$ for 1 hour at $37^{\circ} \mathrm{C}$.

Immunoprecipitation and immunoblotting. These experiments were performed using the antibodies indicated in the figures (23). In general, nephrin was extracted from plasma membranes in RIPA buffer (phosphatebuffered $0.9 \% \mathrm{NaCl}$ containing $0.1 \% \mathrm{SDS}, 1 \% \mathrm{NP}-40$, and $0.5 \%$ sodium deoxycholate). For coimmunoprecipitation experiments of endogenous nephrin and Nck, glomeruli were isolated from kidney cortices of male 
Sprague-Dawley rats (150-200 g) by graded sieving as described previously (52) and lysed by incubation in 1\% NP-40; 20 mM HEPES, pH 7.5; 150 $\mathrm{mM} \mathrm{NaCl}$; and $100 \mathrm{mM}$ potassium iodide with $1 \times$ complete, EDTA-free proteinase inhibitor mixture (Roche Molecular Biochemicals) plus $50 \mathrm{mM}$ $\mathrm{NaF}$ and $1 \mathrm{mM} \mathrm{Na}_{3} \mathrm{VO}_{4}$ at $4{ }^{\circ} \mathrm{C}$ for 30 minutes. Detergent-insoluble material was removed by centrifugation (10,000 $\mathrm{g}$ for 2 minutes).

Mouse kidney perfusion. Perfusion of mouse kidneys with protamine sulfate was carried out essentially as described previously (26). Briefly, adult C57BL/6 mice were anesthetized with pentobarbital; mouse core temperature was monitored with a rectal probe, and animals were maintained at $37^{\circ} \mathrm{C}$ throughout the procedure using a heating pad apparatus. Kidneys were perfused with solutions maintained at $37^{\circ} \mathrm{C}$ through the left ventricle at a pressure of approximately $70 \mathrm{mmHg}$ and an infusion rate of $10 \mathrm{ml} / \mathrm{min}$ (23). Perfusion was carried out with HBSS for 2 minutes followed by perfusion with protamine sulfate $(2 \mathrm{mg} / \mathrm{ml}$ in HBSS; SigmaAldrich) for 15 minutes. For effacement recovery, following perfusion with protamine sulfate, mice were perfused with heparin sulfate $(800$ $\mu \mathrm{g} / \mathrm{ml}$ in HBSS; Sigma-Aldrich) for 15 minutes (26). Kidneys were fixed by perfusion with $3 \%$ paraformaldehyde in PBS before further processing for transmission electron microscopy or indirect immunofluorescence microscopy. All animal experiments were approved by the University Committee on the Use and Care of Animals Institutional Review Board at the University of Michigan Medical School. All work was conducted in accord with the principles and procedures outlined in the National Institutes of Health Guidelines for the Care and Use of Experimental Animals.

Indirect immunofluorescence microscopy. Adult or newborn mouse kidney was fixed in $4 \%$ paraformaldehyde and paraffin embedded. Sections $(4 \mu \mathrm{m})$ were cut, deparaffinized in xylene, and rehydrated through graded alcohols in $\mathrm{H}_{2} \mathrm{O}$. Epitope retrieval was achieved by heating sections at $98^{\circ} \mathrm{C}$ for 2 hours in Retrieve-All-1 (1×; Signet Laboratories Inc.). Sections were cooled to room temperature for 10 minutes, rinsed with water for 1 minute, placed in $1 \times$ PBS for 5 minutes, and made permeable by incubating with $1 \%$ SDS in PBS for 10 minutes. Sections were blocked for 20 minutes in $10 \%$ goat serum prior to overnight incubation of primary antibodies at $4^{\circ} \mathrm{C}$. After 3 washes, secondary antibodies were added at room temperature for 60 minutes. Slides were washed and mounted with a cover slip using ProLong Antifade mounting medium (Invitrogen Corp.). Immunofluorescence images were obtained with a Leica DMIRB inverted microscope and an RT slider digital camera (model 2.3.1; Diagnostic Instruments) and collected with SPOT software (version 4.5; Diagnostic Instruments) and prepared for presentation with Adobe Photoshop version 6.0.

Electron microscopy. Preparation of samples for transmission electron microscopy was performed by standard methods using pieces of diced kidney perfusion fixed in glutaraldehyde/cacodylate buffer. After plastic embedding, $1-\mu \mathrm{m}$ sections were cut and stained with toluidine blue. Selected samples containing glomeruli were thin-sectioned and examined by transmission electron microscopy (23). Twenty glomeruli from each kidney were examined before representative images were chosen.

Glomerular isolation and flotation gradient preparation. Mouse glomeruli were isolated by sieving as described previously (23). The average purity of the mouse glomerular preparations was $70 \%$. Glomeruli were resuspended at a concentration of 10,000 glomeruli/ $\mathrm{ml}$ extraction buffer.
DRM fractions were prepared by flotation gradient centrifugation using Optiprep (Nycomed Pharma AS) as described previously (23). Fyn ${ }^{-/-}$mice (B6;129S-Fyn ${ }^{\text {tm 1Sor }}$ ) obtained from Jackson Laboratory (stock no. 002385) and bred and characterized as previously described were employed in glomerular preparations (23).

Pull-down. Purified GST or GST-nephrinCD was bound to glutathione agarose. Beads were incubated with isolated mouse glomerular lysate extracted with RIPA buffer (phosphate-buffered saline with 1\% NP-40, 0.5\% SDS, and $0.5 \%$ sodium deoxycholate). After washing with PBS containing $0.1 \%$ Tween- $20,1 \mathrm{mM}$ sodium orthovanadate, and $1 \mathrm{mM}$ sodium fluoride, beads were eluted with reduced glutathione. Eluate was resolved by SDSPAGE in replicate prior to immunoblotting with the indicated panel of antibodies (see Table 2).

CD16 chimera Nck recruitment and actin polymerization experiments. NIH 3T3 cells were transfected with CD16 chimeric constructs bearing GFP, HA, or nephrinCD at the C-terminal extent. Thirty hours following transfection, DMEM medium was removed and replaced with fresh medium containing $1 \mu \mathrm{g} / \mathrm{ml}$ CD16 antibody (clone 3G8, catalog no. IM0813; Beckman Coulter). Cells were maintained on ice for 1 hour for Nck recruitment experiments or at $37^{\circ} \mathrm{C}$ for actin experiments. At this point, cells were washed twice with PBS, $1 \mu \mathrm{g} / \mathrm{ml}$ rhodamine-conjugated anti-mouse IgG (Pierce Biotechnology) was added to the media, and incubation was continued at $37^{\circ} \mathrm{C}$ for 20 minutes for recruitment experiment and for 1 hour for actin experiments. Cells were washed 3 times with PBS and fixed with cytoskeleton buffer. The composition of cytoskeleton buffer stock was $10 \mathrm{mM}$ 2-CN-morpholinoethanesulfonic acid, $138 \mathrm{mM} \mathrm{KCl}, 3 \mathrm{mM} \mathrm{MgCl}_{2}, 2 \mathrm{mM}$ EGTA, and sucrose to a final concentration of $0.32 \mathrm{M}$. On the day of use, $20 \%$ paraformaldehyde was added to cytoskeleton buffer stock to achieve a final concentration of $4 \%$. Cover slips were mounted on glass slides using ProLong Gold antifade reagent (Invitrogen Corp.). Samples were analyzed by fluorescence confocal microscopy with an Olympus FV-500 microscope using a $\times 100$ oil immersion objective lens and Fluoroview software (version TIEMPO 4.3; Olympus).

\section{Acknowledgments}

We gratefully acknowledge gifts of reagents from K. Tryggvason (Karolinska Institute), K. Endlich (University of Heidelberg), K. Wary (Texas A\&M University System Health Science Center, Houston, Texas, USA), Y.H. Yakura (Tokyo Metropolitan Institute for Neuroscience, Tokyo, Japan), L. Larose (McGill University, Montreal, Quebec, Canada), and D. Kershaw and B. Margolis (University of Michigan). This work was supported by a Merit Review (to L.B. Holzman) from the Department of Veterans Affairs and by an O'Brien Renal Center Grant from the NIH.

Received for publication November 15, 2005, and accepted in revised form February 7, 2006.

Address correspondence to: Lawrence B. Holzman, University of Michigan Medical School, Division of Nephrology, 1560 Medical Science Research Building II, Ann Arbor, Michigan 481090676, USA. Phone: (734) 764-3157; Fax: (734) 763-0982; E-mail: lholzman@umich.edu.
1. Kerjaschki, D. 2001. Caught flat-footed: podocyte damage and the molecular bases of focal glomerulosclerosis. J. Clin. Invest. 108:1583-1587. doi:10.1172/JCI200114629.

2. Johnstone, D., and Holzman, L.B. 2006. Clinical impact of research on the podocyte slit diaphragm. Nature Clinical Practice Nephrology. In press.

3. Kestila, M., et al. 1998. Positionally cloned gene for a novel glomerular protein--nephrin--is mutat- ed in congenital nephrotic syndrome. Mol. Cell 1:575-582

4. Putaala, H., Soininen, R., Kilpelainen, P., Wartiovaara, J., and Tryggvason, K. 2001. The murine nephrin gene is specifically expressed in kidney, brain and pancreas: inactivation of the gene leads to massive proteinuria and neonatal death. Hum. Mol. Genet. 10:1-8.

5. Hamano, Y., et al. 2002. Determinants of vascular permeability in the kidney glomerulus. J. Biol. Chem. 277:31154-31162.

6. Rantanen, M., et al. 2002. Nephrin TRAP mice lack slit diaphragms and show fibrotic glomeruli and cystic tubular lesions. J. Am. Soc. Nephrol. 13:1586-1594.

7. Holzman, L.B., et al. 1999. Nephrin localizes to the slit pore of the glomerular epithelial cell. Kidney Int. 56:1481-1491. 
8. Ruotsalainen, V., et al. 1999. Nephrin is specifically located at the slit diaphragm of glomerular podocytes. Proc. Natl. Acad. Sci. U. S. A. 96:7962-7967.

9. Shen, K., Fetter, R.D., and Bargmann, C.I. 2004 Synaptic specificity is generated by the synaptic guidepost protein SYG-2 and its receptor, SYG-1. Cell. 116:869-881.

10. Bao, S., and Cagan, R. 2005. Preferential adhesion mediated by Hibris and Roughest regulates morphogenesis and patterning in the Drosophila eye. Dev. Cell. 8:925-935.

11. Shen, K., and Bargmann, C.I. 2003. The immunoglobulin superfamily protein SYG-1 determines the location of specific synapses in C. elegans. Cell. 112:619-630.

12. Benzing, T. 2004. Signaling at the slit diaphragm. J. Am. Soc. Nephrol. 15:1382-1391.

13. Ichimura, K., Kurihara, H., and Sakai, T. 2003. Actin filament organization of foot processes in rat podocytes. J. Histochem. Cytochem. 51:1589-1600.

14. Liu, G., et al. 2003. Neph1 and nephrin interaction in the slit diaphragm is an important determinant of glomerular permeability. J. Clin. Invest. 112:209-221. doi:10.1172/JCI200318242.

15. Shih, N.Y., et al. 1999. Congenital nephrotic syndrome in mice lacking CD2-associated protein. Science. 286:312-315.

16. Lehtonen, S., Lehtonen, E., Kudlicka, K., Holthofer, H., and Farquhar, M.G. 2004. Nephrin forms a complex with adherens junction proteins and CASK in podocytes and in Madin-Darby canine kidney cells expressing nephrin. Am. J. Pathol. 165:923-936.

17. Donoviel, D.B., et al. 2001. Proteinuria and perinatal lethality in mice lacking NEPH1, a novel protein with homology to NEPHRIN. Mol. Cell. Biol. 21:4829-4836.

18. Roselli, S., et al. 2004. Early glomerular filtration defect and severe renal disease in podocin-deficient mice. Mol. Cell. Biol. 24:550-560.

19. Boute, N., et al. 2000. NPHS2, encoding the glomerular protein podocin, is mutated in autosomal recessive steroid-resistant nephrotic syndrome. Nat. Genet. 24:349-354.

20. Li, H., Lemay, S., Aoudjit, L., Kawachi, H., and Takano, T. 2004. SRC-family kinase Fyn phosphorylates the cytoplasmic domain of nephrin and modulates its interaction with podocin. J. Am. Soc. Nephrol. 15:3006-3015.

21. Lahdenpera, J., et al. 2003. Clustering-induced tyrosine phosphorylation of nephrin by Src family kinases. Kidney Int. 64:404-413.

22. Schwarz, K., et al. 2001. Podocin, a raft-associated component of the glomerular slit diaphragm, interacts with CD2AP and nephrin. J. Clin. Invest. 108:1621-1629. doi:10.1172/JCI200112849.

23. Verma, R., et al. 2003. Fyn binds to and phosphorylates the kidney slit diaphragm component Neph- rin. J. Biol. Chem. 278:20716-20723.

24. Simons, K., and Toomre, D. 2000. Lipid rafts and signal transduction. Nat. Rev. Mol. Cell Biol. 1:31-39.

25. Ma, Y.C., Huang, J., Ali, S., Lowry, W., and Huang, X.Y. 2000. Src tyrosine kinase is a novel direct effector of G proteins. Cell. 102:635-646.

26. Reiser, J., et al. 2004. Induction of B7-1 in podocytes is associated with nephrotic syndrome. J. Clin. Invest. 113:1390-1397. doi:10.1172/JCI200420402.

27. Songyang, Z., et al. 1993. SH2 domains recognize specific phosphopeptide sequences. Cell. 72:767-778.

28. Schiwek, D., et al. 2004. Stable expression of nephrin and localization to cell-cell contacts in novel murine podocyte cell lines. Kidney Int. 66:91-101.

29. Kebache, S., Zuo, D., Chevet, E., and Larose, L. 2002. Modulation of protein translation by Nck-1. Proc. Natl. Acad. Sci. U. S. A. 99:5406-5411.

30. Chen, M., She, H., Kim, A., Woodley, D.T., and Li, W. 2000. Nckbeta adapter regulates actin polymerization in NIH 3T3 fibroblasts in response to platelet-derived growth factor bb. Mol. Cell. Biol. 20:7867-7880.

31. Barda-Saad, M., et al. 2005. Dynamic molecular interactions linking the $\mathrm{T}$ cell antigen receptor to the actin cytoskeleton. Nat. Immunol. 6:80-89.

32. Rivera, G.M., Briceno, C.A., Takeshima, F., Snapper, S.B., and Mayer, B.J. 2004. Inducible clustering of membrane-targeted $\mathrm{SH} 3$ domains of the adaptor protein Nck triggers localized actin polymerization. Curr. Biol. 14:11-22.

33. Gerth, V.E., Zhou, X., and Vize, P.D. 2005. Nephrin expression and three-dimensional morphogenesis of the Xenopus pronephric glomus. Dev. Dyn. 233:1131-1139.

34. Kramer-Zucker, A.G., Wiessner, S., Jensen, A.M., and Drummond, I.A. 2005. Organization of the pronephric filtration apparatus in zebrafish requires Nephrin, Podocin and the FERM domain protein Mosaic eyes. Dev. Biol. 285:316-329.

35. Vasioukhin, V., and Fuchs, E. 2001. Actin dynamics and cell-cell adhesion in epithelia. Curr. Opin. Cell Biol. 13:76-84.

36. Vasioukhin, V., Bauer, C., Yin, M., and Fuchs, E. 2000. Directed actin polymerization is the driving force for epithelial cell-cell adhesion. Cell. 100:209-219.

37. Yamaguchi, H., et al. 2005. Molecular mechanisms of invadopodium formation: the role of the $\mathrm{N}$ WASP-Arp $2 / 3$ complex pathway and cofilin. J. Cell Biol. 168:441-452.

38. Gruenheid, S., et al. 2001. Enteropathogenic E. coli Tir binds Nck to initiate actin pedestal formation in host cells. Nat. Cell Biol. 3:856-859.

39. Frischknecht, F., et al. 1999. Actin-based motility of vaccinia virus mimics receptor tyrosine kinase signalling. Nature. 401:926-929.

40. Drees, F., Pokutta, S., Yamada, S., Nelson, W.J., and Weis, W.I. 2005. Alpha-catenin is a molecular switch that binds E-cadherin-beta-catenin and regulates actin-filament assembly. Cell. 123:903-915.

41. Yamada, S., Pokutta, S., Drees, F., Weis, W.I., and Nelson, W.J. 2005. Deconstructing the cadherincatenin-actin complex. Cell. 123:889-901.

42. Simons, M., et al. 2001. Involvement of lipid rafts in nephrin phosphorylation and organization of the glomerular slit diaphragm. Am. J. Pathol. 159:1069-1077.

43. Huber, T.B., et al. 2003. Molecular basis of the functional podocin-nephrin complex: mutations in the NPHS2 gene disrupt nephrin targeting to lipid raft microdomains. Hum. Mol. Genet. 12:3397-3405.

44. Barletta, G.M., Kovari, I.A., Verma, R.K., Kerjaschki, D., and Holzman, L.B. 2003. Nephrin and Neph1 co-localize at the podocyte foot process intercellular junction and form cis hetero-oligomers. J. Biol. Chem. 278:19266-19271.

45. Wartiovaara, J., et al. 2004. Nephrin strands contribute to a porous slit diaphragm scaffold as revealed by electron tomography. J. Clin. Invest. 114:1475-1483. doi:10.1172/JCI200422562.

46. Kebache, S., Cardin, E., Nguyen, D.T., Chevet, E., and Larose, L. 2004. Nck-1 antagonizes the endoplasmic reticulum stress-induced inhibition of translation. J. Biol. Chem. 279:9662-9671.

47. Kershaw, D.B., et al. 1995. Molecular cloning, expression, and characterization of podocalyxinlike protein 1 from rabbit as a transmembrane protein of glomerular podocytes and vascular endothelium. J. Biol. Chem. 270:29439-29446.

48. Mata, M., Merritt, S.E., Fan, G., Yu, G.G., and Holzman, L.B. 1996. Characterization of dual leucine zipper-bearing kinase, a mixed lineage kinase present in synaptic terminals whose phosphorylation state is regulated by membrane depolarization via calcineurin. J. Biol. Chem. 271:16888-16896.

49. Wary, K.K., Mariotti, A., Zurzolo, C., and Giancotti, F.G. 1998. A requirement for caveolin-1 and associated kinase Fyn in integrin signaling and anchorage-dependent cell growth. Cell. 94:625-634.

50. Mizuno, K., et al. 2002. Src homology region 2 domain-containing phosphatase 1 positively regulates B cell receptor-induced apoptosis by modulating association of B cell linker protein with $\mathrm{Nck}$ and activation of c-Jun NH2-terminal kinase. J. Immunol. 169:778-786.

51. Boyle, W.J., van der Geer, P., and Hunter, T. 1991. Phosphopeptide mapping and phosphoamino acid analysis by two-dimentional separation on thin-layer cellulose plates. Methods Enzymol. 201:110-149.

52. Lehtonen, S., et al. 2005. Cell junction-associated proteins IQGAP1, MAGI-2, CASK, spectrins, and alpha-actinin are components of the nephrin multiprotein complex. Proc. Natl. Acad. Sci. U. S. A. 102:9814-9819. 